\title{
Many-body Green's function theory for electron-phonon interactions: the Kadanoff-Baym approach to spectral properties of the Holstein dimer
}

\author{
Niko Säkkinen, ${ }^{1}$ Yang Peng, ${ }^{2,3}$ Heiko Appel, ${ }^{3,4,5}$ and Robert van Leeuwen ${ }^{1,5}$ \\ ${ }^{1)}$ Department of Physics, Nanoscience Center, University of Jyväskylä, Survontie 9, 40014 Jyväskylä, \\ Finland \\ 2) Dahlem Center for Complex Quantum Systems and Fachbereich Physik, Freie Universität Berlin, 14195 Berlin, \\ Germany \\ ${ }^{3)}$ Fritz-Haber-Institut der Max-Planck-Gesellschaft, Faradayweg 4-6, 14195 Berlin-Dahlem, \\ Germany \\ 4) Max-Planck-Institut für Struktur und Dynamik der Materie, Luruper Chaussee 149, 22761 Hamburg, \\ Germany \\ 5) European Theoretical Spectroscopy Facility (ETSF)
}

(Dated: 17 July 2015)

\begin{abstract}
We present a Kadanoff-Baym formalism to study time-dependent phenomena for systems of interacting electrons and phonons in the framework of many-body perturbation theory. The formalism takes correctly into account effects of the initial preparation of an equilibrium state, and allows for an explicit time-dependence of both the electronic and phononic degrees of freedom. The method is applied to investigate the charge neutral and non-neutral excitation spectra of a homogeneous, two-site, two-electron Holstein model. This is an extension of a previous study of the ground state properties in the Hartree (H), partially self-consistent Born (Gd) and fully self-consistent Born (GD) approximations published in Ref. 1. We show that choosing a homogeneous ground state solution leads to unstable dynamics for a sufficiently strong interaction, and that allowing a symmetry-broken state prevents this. The instability is caused by the bifurcation of the ground state and understood physically to be connected with the bipolaronic crossover of the exact system. This mean-field instability persists in the partially self-consistent Born approximation but is not found for the fully self-consistent Born approximation. By understanding the stability properties, we are able to study the linear response regime by calculating the density-density response function by time-propagation. This functions amounts to a solution of the Bethe-Salpeter equation with a sophisticated kernel. The results indicate that none of the approximations is able to describe the respone function during or beyond the bipolaronic crossover for the parameters investigated. In overall, we provide an extensive discussion on when the approximations are valid, and how they fail to describe the studied exact properties of the chosen model system.
\end{abstract}

PACS numbers: 31.15.xm,31.15.xp,71.10.Fd,71.38-k,71.39.Mx,71.15.Qe

\section{INTRODUCTION}

Many-body perturbation theory is one of the most common methodologies used to study quantum transport problems in which interactions among charge carriers or between them and other constituents play a significant role. The method is based on diagrammatic perturbation theory for the non-equilibrium Green's functions together with a set of standard approximations to describe the many-body effects ${ }^{2}$. Although these approximations have been widely used, and thus their properties explored, in the case of steady-state transport ${ }^{3-7}$, much less is known on their performance in the explicitly time-dependent case $^{8-13}$. This is particularly true for systems with moderate to strong electron-phonon interactions in which interesting phenomena like bistability and hysteresis have been observed ${ }^{14}$. As such phenomena are typically driven by many-body interactions, it is natural to ask whether or not the approximate method can describe the relevant physics even qualitatively. This is the case, for example, with the aforementioned bistability whose existence has been subject to doubt on the quality of the method itself ${ }^{15-20}$. The re- cent efforts to realize more sophisticated, but computationally more demanding, approximations have enabled addressing these questions also in the framework of timedependent many-body perturbation theory ${ }^{21-23}$. It is important to study these approximations on a wide scope to understand when they are predictive and the results can be trusted. Time-dependent many-body perturbation theory has also been recently applied to study vibrational effects in ab-initio charge carrier dynamics in semiconductors e.g. relaxation processes after a laser excitation $^{24-26}$. This has become possible as further simplifications, in particular the generalized Kadanoff-Baym Ansatz $^{27}$ (GKBA), have been developed to keep the approach computationally tractable along with the growing system sizes. One could also in this manner study time-dependent phenomena in realistic molecular systems continuing along the lines of the early studies of vibrational effects in photoelectron spectra of molecules ${ }^{28}$ In this context, in order to understand the reasons behind the successes or failures of the methods, it is neccessary to understand the many-body approximations underlying the additional simplifications such as GKBA. There are also topical fields in optoelectronics, such as cavity quantum electrodynamics, and optomechanics in which 
one deals with formally similar systems as in the quantum transport case. Time-dependent many-body perturbation theory has been used in these fields e.g. to derive time-dependent density functionals with preliminary results giving an indication of their quality ${ }^{29,30}$. There is however even less known about the properties of the approximations than in the more established quantum transport setup.

In this work, we present an extension of a previously introduced numerical method ${ }^{31,32}$ to study timedependent, inhomogeneous systems of interacting electrons and phonons. This is also an extension of the equilibrium formalism which we introduced in our earlier work in Ref. 1. Our approach is a variant of time-dependent many-body perturbation theory based on the Kadanoff-Baym equations $(\mathrm{KBE})^{33}$. Here we introduce the relevant equations, time-dependent manybody approximations, and discuss some of their characteristic features e.g. the mean-field, Hartree (H) approximation is shown to lead to the semi-classical Ehrenfest equations. The time-dependent partially ${ }^{34}(\mathrm{Gd})$ and fully $^{22}$ (GD) self-consistent Born approximations are introduced to study correlation effects beyond the meanfield level. These many-body approximations are in particular suited to study time-dependent quantum transport with electron-phonon interactions as they are particle number conserving in the sense of Baym ${ }^{35,36}$. In the present work, the method is instead applied to a finite system since this allows us to assess its performance by comparing the approximate results to an exact solution. Although the method can handle complex timedependent perturbations, we restrict ourselves here to linear response functions obtained by time-propagating the Kadanoff-Baym equations. The density response function $\delta n / \delta v$ obtained in this manner is equivalent to a solution of the Bethe-Salpeter equation (BSE) with an integral kernel which is a functional derivative $\delta \Sigma / \delta G$ of the self-energy $\Sigma$ with respect to the electron propagator $G^{31,37-39}$. The kernel therefore consists of dressed propagators and is fully frequency-dependent in the Born approximations. This level of approximation has, to our best knowledge, not yet been reached in the standard frequency-domain approach even for the much studied purely electronic systems ${ }^{40-43}$. Moreover, as the phonon propagator is determined by the electronic response function, by comparing to the equilibrium phonon propagator we are able to comment on whether or not this additional level of sophistication amounts to improved results. Lastly, we would like to note that although this paper is geared towards electrons and phonons, the method is in fact applicable to a variety of systems of interacting fermions and bosons e.g. electron-photon models $\left(\right.$ Rabi $^{44,45}$ ) and electron-plasmon models (Lundqvist ${ }^{46}$ ).

As the application, we study a homogeneous, two-site, two-electron Holstein model which is a standard model describing interacting electrons and phonons ${ }^{47}$. This continues along the lines of our prior work in Ref. 1 in which we focused on ground-state properties and studying the localizing effect of the electron-phonon interaction by comparing the many-body approximations against exact benchmark results. As a result, we found that the self-energy approximations gave rise to spontaneous symmetry-breaking characterizable by an asymmetric electron density and nuclear displacement. The symmetry-broken solutions as well as solutions obtained by enforcing symmetry were analyzed with the help of total energies, energy components, and natural occupation numbers. It was concluded that the symmetrybreaking can be seen physically to mimic the bipolaronic crossover of the underlying system in which two nearly free electrons form a bound pair with an accompanying nuclear displacement. Moreover, out of the symmetric solutions, only the fully self-consistent Born approximation showed evidence of partially describing this crossover. Here we instead investigate the equilibrium electron and phonon propagators, and linear response functions of the same system using time-dependent many-body perturbation theory. The equilibrium propagators are studied in frequency-domain which gives a more detailed view to the properties of the approximations, and allows us to reevaluate the physical picture obtained from the various energies. The linear response calculations on the other hand allow us to understand better the nature of the symmetric and asymmetric solutions found in our earlier work. In particular, we show that they are equivalent to the equilibrium solutions of the semi-classical equations of the Dicke model ${ }^{48}$ in which the appearance of the asymmetric solution represents the super-radiant phase transition in the thermodynamic limit ${ }^{49-51}$. This transition moreover appears as a bifurcation leading to instability of the symmetric solution which in a finite system is not in agreement with the exact solution. One of the open questions addressed in this work concerns the stability of the symmetric and asymmetric solutions when going beyond the mean-field approximation. In particular, we answer to the question whether or not the symmetric solution retains its stability in the Born approximations. Once stable solutions have been identified, we turn our attention to the linear response functions which are used to assess how the approximations describe the system reacting to a weak perturbation. There is a lot of systematic work on static i.e. zero-frequency susceptibilities of either finite clusters ${ }^{52}$, or extended finite ${ }^{53}$ and infinite $^{54,55}$ dimensional systems with a focus on e.g chargedensity wave phase transition temperatures. Here we thus extend these studies beyond the static case by considering fully frequency-dependent response functions of a finite system.

The paper is organized as follows. In Sec. II, we introduce our method: time-dependent many-body perturbation for electrons interacting with phonons. The method is applied in this work to the model system introduced in Sec. III. The results containing both the equilibrium electron addition and removal, as well as neutral excitation spectra are presented, analyzed and discussed in Sec. IV. The conclusions and an outlook are given in 
Sec. V, and some more technical details are presented in App. A and B.

\section{THEORY}

\section{A. Hamiltonian}

In the present work, we introduce the central concepts of time-dependent many-body perturbation theory for systems of electrons interacting with phonons. Although we do not discuss here electron-electron interactions, they could be included without additional conceptual difficulties. The time-dependent Hamiltonian operator is then given by

$$
\begin{aligned}
\hat{H}(z)= & \sum_{p} \omega_{p}(z) \hat{a}_{p}^{\dagger} \hat{a}_{p}+\sum_{p}\left(f_{p}(z) \hat{a}_{p}^{\dagger}+f_{p}^{*}(z) \hat{a}_{p}\right) \\
& +\sum_{i j} h_{i j}(z) \hat{c}_{i}^{\dagger} \hat{c}_{j} \\
& +\sum_{i j} \sum_{p}\left(m_{j k}^{p}(z) \hat{a}_{p}^{\dagger}+m_{k j}^{p *}(z) \hat{a}_{p}\right) \hat{c}_{j}^{\dagger} \hat{c}_{k},
\end{aligned}
$$

where the properties of the system are encoded in the phonon frequencies $\omega_{p}$, generalized forces $f_{p}$, elements of the electron one-body Hamiltonian $h_{i j}$, and electronphonon interaction elements $m_{i j}^{p}$.

These quantities all depend on a time-argument lying on the extended Keldysh contour ${ }^{2}$ shown in Fig. 1. The Hamiltonian operator is however the same on the forward $(-)$ and backward $(+)$ branches, and independent of the contour time on the vertical equilibrium $(M)$ track as in our prior work in Ref. 1. An explicit timedependence allows one to realize a variety of physical scenarios from electrons and nuclei driven by electromagnetic fields to more abstract simulations based on an interaction quench. In the present work, we however focus on another type of time-dependence arising from the choice of the initial state.

The electrons and phonons are described in second quantization with annihilation $\hat{c}_{i}, \hat{a}_{p}$ and creation $\hat{c}_{i}^{\dagger}, \hat{a}_{p}^{\dagger}$ operators which obey canonical anti-commutation and commutation relations, respectively. In order to facilitate a compact presentation of the many-body perturbation theory, we further introduce the self-adjoint phonon operators

$$
\hat{\phi}_{1, p} \equiv\left(\hat{a}_{p}^{\dagger}+\hat{a}_{p}\right) / \sqrt{2}, \quad \hat{\phi}_{2, p} \equiv \imath\left(\hat{a}_{p}^{\dagger}-\hat{a}_{p}\right) / \sqrt{2},
$$

to which we associate a collective index $P \equiv\left\{\varsigma_{p} \in\right.$ $\{1,2\}, p\}$ so that we can write their commutation relation compactly as

$$
\left[\hat{\phi}_{P}, \hat{\phi}_{Q}\right]=\alpha_{P Q}
$$

where $\alpha_{1 p, 1 q}=\alpha_{2 p, 2 q}=0$ and $\alpha_{1 p, 2 q}=-\alpha_{2 q, 1 p}=\imath \delta_{p q}$. These operators can be physically understood as components of the displacement $\left(\hat{\phi}_{1 p}\right)$ and momentum $\left(\hat{\phi}_{2 p}\right)$

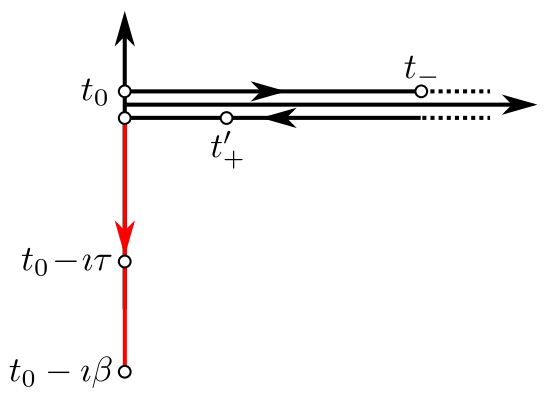

FIG. 1. The extended Keldysh contour which consists of the vertical, imaginary-time track responsible for the initial equilibrium preparation, and of the horizontal forward (-) and backward $(+)$ real-time tracks related to the real-time time-evolution. (color online)

operators. They allow us to rewrite the Hamiltonian operator as

$$
\begin{aligned}
\hat{H}(z)= & \sum_{P Q} \Omega_{P Q}(z) \hat{\phi}_{P} \hat{\phi}_{Q}+\sum_{P} F_{P}(z) \hat{\phi}_{P} \\
& +\sum_{i j} h_{i j}(z) \hat{c}_{i}^{\dagger} \hat{c}_{j} \\
& +\sum_{i j} \sum_{P} M_{i j}^{P}(z) \hat{\phi}_{P} \hat{c}_{i}^{\dagger} \hat{c}_{j}
\end{aligned}
$$

where the phonon frequencies, generalized forces, and electron-phonon interaction are incorporated into

$$
\begin{aligned}
F_{p \varsigma_{p}}(z) & \equiv \delta_{\varsigma_{p}, 1}\left(f_{p}(z)+f_{p}^{*}(z)\right) / \sqrt{2} \\
& -\imath \delta_{\varsigma_{p}, 2}\left(f_{p}(z)-f_{p}^{*}(z)\right) / \sqrt{2}, \\
\Omega_{p \varsigma_{p}, q \varsigma_{q}}(z) & \equiv \omega_{p}(z)\left(\delta_{p q} \delta_{\varsigma_{p} \varsigma_{q}}+\alpha_{p \varsigma_{p}, q \varsigma_{q}}\right) / 2, \\
M_{j k}^{p \varsigma_{p}}(z) & \equiv \delta_{\varsigma_{p}, 1}\left(m_{j k}^{p}(z)+m_{k j}^{p *}(z)\right) / \sqrt{2} \\
& -\imath \delta_{\varsigma_{p}, 2}\left(m_{j k}^{p}(z)-m_{k j}^{p *}(z)\right) / \sqrt{2},
\end{aligned}
$$

which are to be understood in this work to represent elements of a vector, matrix, and a vector of matrices, respectively. The one-body electron Hamiltonian elements are also to be understood as elements of a matrix. In the following an overhead arrow denotes a vector $(\vec{F})$, boldfaced symbols matrices $(\boldsymbol{\Omega}, \boldsymbol{h})$, and a combination of these two a vector of matrices $(\overrightarrow{\boldsymbol{M}})$, while tr denotes a matrix trace.

\section{B. Many-Body Perturbation Theory}

The central quantities of many-body perturbation theory of interacting electrons and phonons are the phonon field expectation value, and the phonon and electron 
propagators defined as

$$
\begin{aligned}
\phi_{P}(z) & \equiv \frac{1}{\mathcal{Z}} \operatorname{Tr}\left[\mathcal{T}\left\{e^{-\imath \int_{C} d \bar{z} \hat{H}(\bar{z})} \hat{\phi}_{P}(z)\right\}\right] \\
D_{P Q}\left(z ; z^{\prime}\right) & \equiv \frac{1}{\imath \mathcal{Z}} \operatorname{Tr}\left[\mathcal{T}\left\{e^{-\imath \int_{C} d \bar{z} H(\bar{z})} \Delta \hat{\phi}_{P}(z) \Delta \hat{\phi}_{Q}\left(z^{\prime}\right)\right\}\right] \\
G_{i j}\left(z ; z^{\prime}\right) & \equiv \frac{1}{\imath \mathcal{Z}} \operatorname{Tr}\left[\mathcal{T}\left\{e^{-\imath \int_{C} d \bar{z} H(\bar{z})} \hat{c}_{i}(z) \hat{c}_{j}^{\dagger}\left(z^{\prime}\right)\right\}\right]
\end{aligned}
$$

where $\Delta \hat{\phi}_{P} \equiv \hat{\phi}_{P}-\phi_{P}$ is a fluctuation operator, $\mathcal{Z} \equiv$ $\operatorname{Tr}\left[e^{-\imath \int d z \hat{H}(z)}\right]$ the partition function, $\operatorname{Tr}$ the trace over a complete set of quantum states, and $\mathcal{T}$ is the timeordering operator on a Keldysh time-contour $C$ of Fig. 1 acting on operators given in the Schrödinger picture but having time-arguments $z, z^{\prime}$ for book-keeping reasons ${ }^{2}$. These objects have a closed form perturbation expansion obtained using Wick's theorem and re-summing all terms into two electron and phonon propagator line irreducible contributions. This leads to the equations

$$
\begin{gathered}
\vec{\phi}(z)=\int_{C} d \bar{z} \boldsymbol{d}(z ; \bar{z})\left(\vec{F}(\bar{z})-\imath \operatorname{tr}\left(\overrightarrow{\boldsymbol{M}}(\bar{z}) \boldsymbol{G}\left(\bar{z} ; \bar{z}^{+}\right)\right)\right. \\
\boldsymbol{D}\left(z ; z^{\prime}\right)=\boldsymbol{d}\left(z ; z^{\prime}\right)+\int_{C} d \bar{z} d \bar{z}^{\prime} \boldsymbol{d}(z ; \bar{z}) \boldsymbol{\Pi}\left(\bar{z} ; \bar{z}^{\prime}\right) \boldsymbol{D}\left(\bar{z}^{\prime} ; z^{\prime}\right) \\
\boldsymbol{G}\left(z ; z^{\prime}\right)=\boldsymbol{g}\left(z ; z^{\prime}\right)+\int_{C} d \bar{z} d \bar{z}^{\prime} \boldsymbol{g}(z ; \bar{z}) \boldsymbol{\Sigma}\left(\bar{z} ; \bar{z}^{\prime}\right) \boldsymbol{G}\left(\bar{z}^{\prime} ; z^{\prime}\right)
\end{gathered}
$$

where $g$ and $d$ denote the non-interacting electron and phonon propagators defined by Eqs. (3) and (2) in the absence of the electron-phonon interaction. The integral kernels $\Sigma \equiv \Sigma[G, D]$ and $\Pi \equiv \Pi[G, D]$ are non-local one-body potentials known as electron and phonon selfenergies. These self-energies contain information on interactions of the system, as well as the external driving induced by the generalized force $F$. The non-interacting electron and phonon propagators are given respectively by

$$
\begin{aligned}
& \boldsymbol{g}\left(z ; z^{\prime}\right)=-\imath \boldsymbol{U}\left(z, t_{0}\right)\left(\boldsymbol{\theta}\left(z, z^{\prime}\right)-\boldsymbol{f}_{+}\left(\beta \boldsymbol{h}^{M}\right)\right) \boldsymbol{U}\left(t_{0}, z^{\prime}\right) \\
& \boldsymbol{d}\left(z ; z^{\prime}\right)=-\imath \boldsymbol{\alpha} \boldsymbol{V}\left(z, t_{0}\right)\left(\boldsymbol{\theta}\left(z, z^{\prime}\right)+\boldsymbol{f}_{-}\left(\beta \tilde{\boldsymbol{\Omega}}^{M} \boldsymbol{\alpha}\right)\right) \boldsymbol{V}\left(t_{0}, z^{\prime}\right)
\end{aligned}
$$

where $\boldsymbol{\theta} \equiv \theta \mathbf{1}$ with $\theta$ being the Heaviside function and 1 the identity matrix, $\beta$ is the inverse temperature, $f_{ \pm}$ denote the Fermi-Dirac $(+)$ and Bose-Einstein $(-)$ distribution functions, $\tilde{\boldsymbol{\Omega}}^{M} \equiv \tilde{\boldsymbol{\Omega}}\left(t_{0}-\imath \tau\right)$ independent of $\tau$ is the Matsubara component of

$$
\tilde{\boldsymbol{\Omega}}(z) \equiv \boldsymbol{\Omega}(z)+\boldsymbol{\Omega}^{T}(z) .
$$

Finally, we introduced the time-evolution matrices as solutions to

$$
\begin{aligned}
\imath \partial_{z} \boldsymbol{U}\left(z, z^{\prime}\right) & =\boldsymbol{h}(z) \boldsymbol{U}\left(z, z^{\prime}\right), \\
-\imath \partial_{z^{\prime}} \boldsymbol{U}\left(z, z^{\prime}\right) & =\boldsymbol{U}\left(z, z^{\prime}\right) \boldsymbol{h}\left(z^{\prime}\right), \\
\imath \partial_{z} \boldsymbol{V}\left(z, z^{\prime}\right) & =\tilde{\boldsymbol{\Omega}}(z) \boldsymbol{\alpha} \boldsymbol{V}\left(z, z^{\prime}\right), \\
-\imath \partial_{z^{\prime}} \boldsymbol{V}\left(z, z^{\prime}\right) & =\boldsymbol{V}\left(z, z^{\prime}\right) \tilde{\boldsymbol{\Omega}}(z) \boldsymbol{\alpha},
\end{aligned}
$$

with the initial conditions $\boldsymbol{U}\left(t_{0}, t_{0}\right)=\boldsymbol{V}\left(t_{0}, t_{0}\right)=\mathbf{1}$.

In our earlier work in Ref. 1, we introduced our implementation of the equilibrium Matsubara formalism obtained by choosing time-arguments $z=t_{0}-\imath \tau, z^{\prime}=$ $t_{0}-\imath \tau^{\prime}$ on the imaginary track. Here we focus on an extension of this formalism to time-dependent cases in which it is more natural to differentiate Eqs. (4) with respect to the first contour time in order to arrive at the equations of motion

$$
\begin{aligned}
& \left(\imath \boldsymbol{\alpha} \partial_{z}-\tilde{\boldsymbol{\Omega}}(z)\right) \vec{\phi}(z) \\
& =\vec{F}(z)-\imath \operatorname{tr}\left(\overrightarrow{\boldsymbol{M}}(z) \boldsymbol{G}\left(z ; z^{+}\right)\right) \\
& \left(\imath \boldsymbol{\alpha} \partial_{z}-\tilde{\boldsymbol{\Omega}}(z)\right) \boldsymbol{D}\left(z ; z^{\prime}\right) \\
& =\boldsymbol{\delta}\left(z, z^{\prime}\right)+\int_{C} d z \boldsymbol{\Pi}(z ; \bar{z}) \boldsymbol{D}\left(\bar{z} ; z^{\prime}\right) \\
& \left(\boldsymbol{\imath} \partial_{z}-\boldsymbol{h}(z)\right) \boldsymbol{G}\left(z ; z^{\prime}\right) \\
& =\boldsymbol{\delta}\left(z, z^{\prime}\right)+\int_{C} d z \boldsymbol{\Sigma}(z ; \bar{z}) \boldsymbol{G}\left(\bar{z} ; z^{\prime}\right)
\end{aligned}
$$

where $\boldsymbol{\imath}=\imath \mathbf{1}$. These equations together with their conjugate equations obtained by differentiating with respect to the second time-argument of the propagators form a closed set of the equations which can be solved once an approximation for the many-body part of the self-energy has been fixed.

\section{Self-Energies}

The self-energy $\Sigma$, as noted above, contains both a contribution arising from the generalized force $F_{P}(z)$, as well as a part induced by the electron-phonon interactions. The phonon propagator, being defined in terms of fluctuation operators, is not directly influenced by this force, instead it appears in the electron self-energy and can be handled by writing the self-energy as

$$
\Sigma_{i j}\left(z ; z^{\prime}\right)=\delta\left(z, z^{\prime}\right) v_{n, i j}(z)+\Sigma_{\mathrm{MB}, i j}\left(z ; z^{\prime}\right)
$$

where we introduced the potential

$$
v_{n, i j}(z) \equiv \sum_{P Q} M_{i j}^{P}(z) \int_{C} d \bar{z} d_{P Q}(z, \bar{z}) F_{Q}(\bar{z})
$$

which represents the classical potential induced by nuclei experiencing a generalized force $F_{Q}$. The many-body self-energy, denoted by MB, is then subject to approximation. The approximations used here, and introduced 
earlier in Ref. 1, are summarized diagrammatically in Fig. 2. The approximate electron self-energies consists of the Hartree (H) and Fock (F) diagrams. The Hartree diagram can be written as

$$
\Sigma_{\mathrm{H}}[G]_{i j}\left(z ; z^{\prime}\right)=\delta\left(z, z^{\prime}\right) v_{\mathrm{H}}[G]_{i j}(z),
$$

where the time-local Hartree potential is given by

$$
\begin{aligned}
v_{\mathrm{H}}[G]_{i j}(z) & =-\imath \sum_{\substack{k l \\
P Q}} M_{i j}^{P}(z) \\
& \times \int_{C} d \bar{z} d_{P Q}(z, \bar{z}) M_{k l}^{Q}(\bar{z}) G_{l k}\left(\bar{z} ; \bar{z}^{+}\right) .
\end{aligned}
$$

or alternatively by

$$
v_{\mathrm{H}}[G]_{i j}(z)=\sum_{P} M_{i j}^{P}(z) \phi_{P}(z)-v_{n, i j}(z),
$$

which follows from the equation of motion for the noninteracting phonon propagator. Electron self-energy terms beyond Hartree contribute to the exchangecorrelation, many-body self-energy

$$
\Sigma_{\mathrm{xc}, i j}\left(z ; z^{\prime}\right) \equiv \Sigma_{\mathrm{MB}, i j}\left(z ; z^{\prime}\right)-\Sigma_{\mathrm{H}, i j}\left(z ; z^{\prime}\right),
$$

whose lowest order diagram is the Fock diagram

$$
\begin{aligned}
& \Sigma_{\mathrm{F}}[G, D]_{i j}\left(z ; z^{\prime}\right) \\
& \quad=\imath \sum_{k l, P Q} M_{i k}^{P}(z) M_{l j}^{Q}\left(z^{\prime}\right) D_{P Q}\left(z ; z^{\prime}\right) G_{k l}\left(z ; z^{\prime}\right),
\end{aligned}
$$

which is a time-nonlocal memory term describing singlephonon absorption/emission processes. The only phonon self-energy diagram used in this work is the bubble diagram

$$
\begin{aligned}
& \Pi_{\mathrm{B}}[G]_{P Q}\left(z ; z^{\prime}\right) \\
& \quad=-\imath \sum_{i j, k l} M_{i j}^{P}(z) M_{k l}^{Q}\left(z^{\prime}\right) G_{l i}\left(z^{\prime} ; z\right) G_{j k}\left(z ; z^{\prime}\right),
\end{aligned}
$$

which describes simple phonon induced electron-hole excitation processes.

The many-body self-energies and their abbreviations used throughout the text are summarized in the list below.

H: The Hartree approximation consists of approximating the electron self-energy with the Hartree diagram

$$
\Sigma_{\mathrm{H}}[G]_{i j}\left(z ; z^{\prime}\right)=\delta\left(z, z^{\prime}\right) v_{\mathrm{H}}[G]_{i j}(z),
$$

and neglecting the phonon self-energy. This is a mean-field approximation in which electrons feel only the classical potential due to nuclei. The resulting Hartree equations

$$
\begin{aligned}
\imath \frac{d}{d z} \boldsymbol{G}\left(z ; z^{+}\right) & =\left[\boldsymbol{h}(z)+\boldsymbol{v}_{n}(z)\right. \\
& \left.+\boldsymbol{v}_{\mathrm{H}}(z), \boldsymbol{G}\left(z ; z^{+}\right)\right], \\
\imath \boldsymbol{\alpha} \partial_{z} \vec{\phi}(z) & =\tilde{\boldsymbol{\Omega}}(z) \vec{\phi}(z)+\vec{F}(z) \\
& -\imath \operatorname{tr}\left(\overrightarrow{\boldsymbol{M}} \boldsymbol{G}\left(z ; z^{+}\right)\right),
\end{aligned}
$$

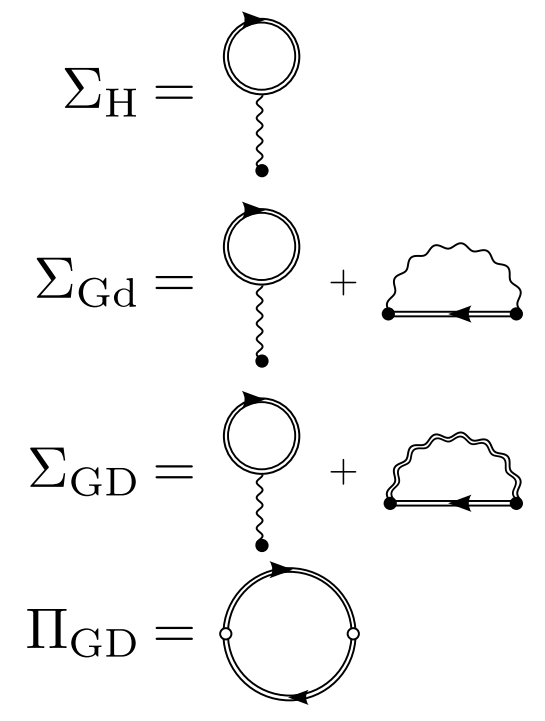

FIG. 2. The Hartree (H), partially self-consistent (Gd), and fully self-consistent (GD) Born self-energies summarize the many-body approximations used in this work. A two-fold line with an arrow indicates a dressed electron propagator, while single and two-fold wiggly lines represent bare and dressed phonon propagators, respectively. An open circle and a closed circle represent a connection for a phonon and an electron propagator, respectively.

can be shown to be equivalent to the semi-classical Ehrenfest equations, see App. A.

Gd: The partially self-consistent Born approximation amounts to approximating the electron many-body self-energy with

$$
\begin{aligned}
\Sigma_{\mathrm{Gd}}[G]_{i j}\left(z ; z^{\prime}\right) & \equiv \Sigma_{\mathrm{H}}[G]_{i j}\left(z ; z^{\prime}\right) \\
& +\Sigma_{\mathrm{F}}[G, d]_{i j}\left(z ; z^{\prime}\right)
\end{aligned}
$$

where $d$ is the bare phonon propagator obtained by putting the phonon self-energy to zero. This amounts to saying that that the nuclei are unaffected by the electronic particle-hole excitations.

GD: The fully self-consistent Born approximation is defined by writing the electron many-body self-energy as

$$
\begin{aligned}
\Sigma_{\mathrm{GD}}[G, D]_{i j}\left(z ; z^{\prime}\right) & \equiv \Sigma_{\mathrm{H}}[G]_{i j}\left(z ; z^{\prime}\right) \\
& +\Sigma_{\mathrm{F}}[G, D]_{i j}\left(z ; z^{\prime}\right)
\end{aligned}
$$

while the phonon self-energy is given by

$$
\Pi_{\mathrm{GD}}[G]_{P Q}\left(z ; z^{\prime}\right) \equiv \Pi_{\mathrm{B}}[G]_{P Q}\left(z ; z^{\prime}\right) .
$$

Note that although we dress the phonon propagator in the Fock diagram, one should not use a dressed propagator in the Hartree diagram as it leads to double-counting of terms in the perturbation expansion $^{2,56}$. 


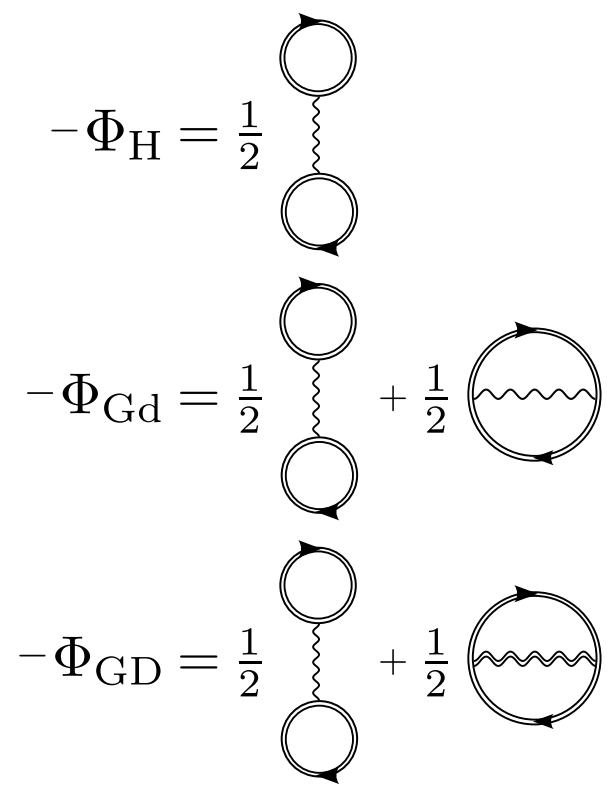

FIG. 3. The $\Phi$-functionals for the Hartree (H), partially selfconsistent (Gd), and fully self-consistent (GD) Born approximations. A two-fold line with an arrow indicates a dressed electron propagator, while single and two-fold wiggly lines represent bare and dressed phonon propagators, respectively. Note that the minus sign on the left hand side arises due to the loop rule ${ }^{2}$.

These approximations are all $\Phi$-derivable, that is the corresponding self-energies can be obtained as the functional derivatives

$$
\begin{aligned}
\Sigma_{i j}[G, D]\left(z ; z^{\prime}\right) & =\frac{\delta \Phi[G, D]}{\delta G_{j i}\left(z^{\prime} ; z\right)}, \\
\Pi_{P Q}[G, D]\left(z ; z^{\prime}\right) & =-\left.2 \frac{\delta \Phi[G, D]}{\delta D_{Q P}\left(z^{\prime} ; z\right)}\right|_{S},
\end{aligned}
$$

of an approximate $\Phi$-functional which are shown in Fig. 3. Note that the subscript $S$ refers to a symmetrized functional derivative, $\mathrm{see}^{2}$. The $\Phi$-derivability of the electron self-energy together with self-consistency in the electron propagator guarantee gauge invariance and consequently fulfillment of the electron density conservation $\operatorname{law}^{35,36}$.

\section{Kadanoff-Baym Equations}

The equations of motion of Eqs. (5) are customary solved by projecting the propagators to different parts of the Keldysh contour by choosing the time-arguments appropriately $^{2}$. This procedure leads to the greater $(>)$, lesser $(<)$, left $(\lceil)$, right (\rceil$)$, and Matsubara $(M)$ com- ponents

$$
\begin{aligned}
a^{\gtrless}\left(t ; t^{\prime}\right) & \equiv a\left(t_{ \pm} ; t_{\mp}^{\prime}\right), \\
a^{\rceil}(t ; \tau) & \equiv a\left(t ; t_{0}-\imath \tau\right), \\
a^{\Gamma}(\tau ; t) & \equiv a\left(t_{0}-\imath \tau ; t\right), \\
a^{M}\left(\tau ; \tau^{\prime}\right) & \equiv a\left(t_{0}-\imath \tau ; t_{0}-\imath \tau^{\prime}\right),
\end{aligned}
$$

where the subscript $\mp$ denotes a time evaluated on the forward/backward branches of the contour, and $a$ is a function in the space of Keldysh functions ${ }^{2}$. The Keldysh components of the phonon and electron propagator obey the symmetries

$$
\begin{aligned}
G_{i j}^{\gtrless}\left(t ; t^{\prime}\right) & =-\left[G_{j i}^{\gtrless}\left(t^{\prime} ; t\right)\right]^{*}, \\
G_{i j}^{\Gamma}(\tau ; t) & =\left[G_{j i}^{\rceil}(t ; \beta-\tau)\right]^{*}, \\
D_{P Q}^{\gtrless}\left(t ; t^{\prime}\right) & =-\left[D_{Q P}^{\gtrless}\left(t^{\prime} ; t\right)\right]^{*}=-\left[D_{P Q}^{\lessgtr}\left(t ; t^{\prime}\right)\right]^{*}, \\
D_{P Q}^{\Gamma}(\tau ; t) & =\left[D_{Q P}^{\rceil}(t ; \beta-\tau)\right]^{*}=\left[D_{P Q}^{\Gamma}(\beta-\tau ; t)\right]^{*},
\end{aligned}
$$

where the additional symmetries of the phonon propagator are due to the symmetry $D_{P Q}\left(z ; z^{\prime}\right)=D_{Q P}\left(z^{\prime}, z\right)$. The equations of motion obtained by taking all possible components of the contour-time equations of motion form a set of non-linear integro-differential equations of motion known as the Kadanoff-Baym equations ${ }^{33}$. The symmetries of the propagator however imply that we only need equations of motion for the greater, lesser, and right components where the first two are required for times $t \geq t^{\prime}$. The relevant equations of motion are then

$$
\begin{aligned}
\imath \partial_{t} \boldsymbol{G}^{\gtrless}\left(t ; t^{\prime}\right) & =\boldsymbol{h}_{\mathrm{eff}}(t) \boldsymbol{G}^{\gtrless}\left(t ; t^{\prime}\right)+\boldsymbol{I}^{\gtrless}\left[\Sigma_{\mathrm{xc}}, G\right]\left(t ; t^{\prime}\right), \\
\imath \partial_{t} \boldsymbol{G}^{\boldsymbol{\top}}(t ; \tau) & =\boldsymbol{h}_{\mathrm{eff}}(t) \boldsymbol{G}^{\rceil}(t ; \tau)+\boldsymbol{I}^{\rceil}\left[\Sigma_{\mathrm{xc}}, G\right]\left(t ; t^{\prime}\right), \\
\imath \partial_{t} \boldsymbol{D}^{\gtrless}\left(t ; t^{\prime}\right) & =\boldsymbol{\alpha}\left(\tilde{\boldsymbol{\Omega}}(t) \boldsymbol{D}^{\gtrless}\left(t ; t^{\prime}\right)+\boldsymbol{I}^{\gtrless}[\Pi, D]\left(t ; t^{\prime}\right)\right), \\
\imath \partial_{t} \boldsymbol{D}^{\rceil}(t ; \tau) & =\boldsymbol{\alpha}\left(\tilde{\boldsymbol{\Omega}}(t) \boldsymbol{D}^{\rceil}(t ; \tau)+\boldsymbol{I}^{\rceil}[\Pi, D](t ; \tau)\right),
\end{aligned}
$$

for off time-diagonal and

$$
\begin{aligned}
& \imath \frac{d}{d t} \boldsymbol{G}^{\gtrless}(t ; t)=\boldsymbol{h}_{\mathrm{eff}}(t) \boldsymbol{G}^{\gtrless}(t ; t)+\boldsymbol{I}^{\gtrless}\left[\Sigma_{\mathrm{xc}}, G\right](t ; t)+\text { h.c. }, \\
& \imath \frac{d}{d t} \boldsymbol{D}^{\gtrless}(t ; t)=\boldsymbol{\alpha}\left(\tilde{\boldsymbol{\Omega}}(t) \boldsymbol{D}^{\gtrless}(t ; t)+\boldsymbol{I}^{\gtrless}[\Pi, D](t ; t)\right)+\text { h.c. },
\end{aligned}
$$

where h.c. denotes the Hermitian conjugate, for on timediagonal time-propagation. Here we introduced the effective one-body electron Hamiltonian

$$
\boldsymbol{h}_{\mathrm{eff}}(t) \equiv \boldsymbol{h}(t)+\boldsymbol{v}_{n}(t)+\boldsymbol{v}_{\mathrm{H}}(t),
$$

as well as the collision integrals

$$
\begin{aligned}
I^{\gtrless}[a, b]\left(t ; t^{\prime}\right) & \equiv\left[a^{\rceil} \star b^{\lceil}\right]\left(t ; t^{\prime}\right) \\
& +\left[a^{R} \bullet b^{\gtrless}\right]\left(t ; t^{\prime}\right)+\left[a^{\gtrless} \bullet b^{A}\right]\left(t ; t^{\prime}\right), \\
I^{\urcorner}[a, b](t ; \tau) & \left.\equiv[a\rceil \star b^{M}\right](t ; \tau)+\left[a^{R} \bullet b^{\rceil}\right](t ; \tau) .
\end{aligned}
$$


with the bullets and stars denoting convolution integrals of the form

$$
\begin{aligned}
& {[a \bullet b]\left(t ; t^{\prime}\right)=\int_{t_{0}}^{\infty} d \bar{t} a(t, \bar{t}) b\left(\bar{t}, t^{\prime}\right),} \\
& {[a \star b]\left(t ; t^{\prime}\right)=-\imath \int_{0}^{\beta} d \tau a(t, \tau) b\left(\tau, t^{\prime}\right),}
\end{aligned}
$$

where $a$ and $b$ are possibly matrix valued functions on the Keldysh contour. The Hartree potential appearing in the effective Hamiltonian can be evaluated using Eq. (9) instead of Eq. (8) by taking advantage of the equation of motion

$$
\begin{aligned}
\imath \partial_{t} \vec{\phi}(t) & =\boldsymbol{\alpha}(\tilde{\boldsymbol{\Omega}}(t) \vec{\phi}(t) \\
& \left.+\vec{F}(t)-\imath \operatorname{tr}\left(\overrightarrow{\boldsymbol{M}}(t) \boldsymbol{G}^{<}(t ; t)\right)\right),
\end{aligned}
$$

for the real-time $\phi_{P}(t) \equiv \phi_{P}\left(t_{ \pm}\right)$phonon field expectation value. The Kadanoff-Baym equations, including the equation above, then form a closed set of equations which, when supplemented with the initial conditions

$$
\begin{aligned}
G_{i j}^{\gtrless}\left(t_{0} ; t_{0}\right) & =G_{i j}^{M}\left(0^{ \pm}\right), \\
G_{i j}^{\rceil}\left(t_{0} ; \tau\right) & =G_{i j}^{M}(-\tau), \\
D_{P Q}^{\gtrless}\left(t_{0} ; t_{0}\right) & =D_{P Q}^{M}\left(0^{ \pm}\right), \\
D_{P Q}^{\rceil}\left(t_{0} ; \tau\right) & =D_{P Q}^{M}(-\tau), \\
\phi_{P}\left(t_{0}\right) & =\phi_{P}^{M},
\end{aligned}
$$

given by the equilibrium Matsubara components introduced in Ref. 1, can be solved on a computer by time propagation $^{32}$.

\section{MODEL}

Our model system is a two-site Holstein model ${ }^{57-69}$ which can be viewed as a minimal representation of a system in which electrons move between two molecules, so that they are coupled to the local vibrational modes of these molecules. In the case of two identical molecules, we find that only the relative displacement couples to the electron density difference between the molecules, and thus the Hamiltonian operator for the isolated system reduces to

$$
\begin{aligned}
\hat{H}^{M} & \equiv \omega_{0} \hat{a}^{\dagger} \hat{a} \\
& -t_{\text {kin }} \sum_{\sigma}\left(\hat{c}_{1 \sigma}^{\dagger} \hat{c}_{2 \sigma}+\hat{c}_{2 \sigma}^{\dagger} \hat{c}_{1 \sigma}\right) \\
& -\frac{g}{\sqrt{2}}\left(\hat{a}^{\dagger}+\hat{a}\right) \sum_{\sigma}\left(\hat{n}_{1 \sigma}-\hat{n}_{2 \sigma}\right),
\end{aligned}
$$

where $\hat{a}$ and $\hat{a}^{\dagger}$ annihilate and create a phonon to the relative displacement mode, $\hat{c}_{i \sigma}$ is the electronic operator that annihilates an electron of spin $\sigma$ at site $i$, and $\hat{n}_{i \sigma} \equiv \hat{c}_{i \sigma}^{\dagger} \hat{c}_{i \sigma}$ is the electron density operator at site $i$.
The parameters $\omega_{0}, t_{\text {kin }}$ and $g$ characterize the bare vibrational frequency, inter-site hopping and local electronphonon interaction strength, respectively. This Hamiltonian corresponds to the relative Hamiltonian of Ref. 1 which is chosen here over the full Hamiltonian due to both its simplicity and computational reasons. The system can be probed with external time-dependent fields which are described with the Hamiltonian operator

$$
\hat{H}(t) \equiv \hat{H}^{M}+f(t)\left(\hat{a}^{\dagger}+\hat{a}\right)+\sum_{i \sigma} v_{i}(t) \hat{n}_{i \sigma}
$$

where $f$ and $v_{i}$ describe amplitudes of the external fields acting on the nuclei and electrons, respectively. The displacement and momentum operators, defined in this model as $\hat{u} \equiv\left(\hat{a}^{\dagger}+\hat{a}\right) / \sqrt{2}$ and $\hat{p} \equiv \imath\left(\hat{a}^{\dagger}-\hat{a}\right) / \sqrt{2}$, allow us to rewrite the Hamiltonian operator as

$$
\begin{aligned}
\hat{H}(t)= & \hat{H}^{M}+\sqrt{2} f(t) \hat{u}+\sum_{i \sigma} v_{i}(t) \hat{n}_{i \sigma} \\
\hat{H}^{M}= & \frac{\omega_{0}}{2}\left(\hat{p}^{2}+\hat{u}^{2}-1\right) \\
& -t_{\mathrm{kin}} \sum_{\sigma}\left(\hat{c}_{1 \sigma}^{\dagger} \hat{c}_{2 \sigma}+\hat{c}_{2 \sigma}^{\dagger} \hat{c}_{1 \sigma}\right) \\
& -g \hat{u} \sum_{\sigma}\left(\hat{n}_{1 \sigma}-\hat{n}_{2 \sigma}\right)
\end{aligned}
$$

which is equivalent to the Hamiltonian of Eq. (1) with the matrix elements

$$
\begin{aligned}
F_{\varsigma_{p}}(z) & =\theta\left(t_{0+}, z\right) \delta_{\varsigma_{i}, 1} \sqrt{2} f(z), \\
\Omega_{\varsigma_{p}, \varsigma_{q}}(z) & =\omega_{0}\left(\delta_{\varsigma_{p} \varsigma_{q}}+\alpha_{\varsigma_{p} \varsigma_{q}}\right) / 2, \\
M_{i \sigma, j \sigma^{\prime}}^{\varsigma_{p}}(z) & =-g \delta_{\varsigma_{p}, 1} \delta_{\sigma \sigma^{\prime}} \delta_{i j}\left(\delta_{i 1}-\delta_{i 2}\right),
\end{aligned}
$$

where we dropped the phonon mode index due to having only the relative mode. Moreover, here $\theta$ denotes a contour-time Heaviside function and $t_{0+}$ the origin of the backward branch. The time-independent properties of this model depend on the two dimensionless parameters

$$
\begin{aligned}
\gamma & \equiv \frac{\omega_{0}}{t_{\text {kin }}}, \\
\lambda & \equiv \frac{2 g^{2}}{t_{\text {kin }} \omega_{0}}
\end{aligned}
$$

denoting the adiabatic ratio and effective interaction. The adiabatic ratio $\gamma$ describes the relative energy scale of electrons and nuclei, while the effective interaction $\lambda$ is a measure of the coupling between the motions of these two constituents.

\section{RESULTS}

In the following, we present our results for the equilibrium propagators and linear response functions. The results are for a system initially at zero temperature in the pure two-electron $N=2$ spin singlet $S^{2}=S_{z}=0$ 
ground state. This is mimicked in many-body perturbation theory with the inverse temperature $\beta / \omega_{0}^{-1}=10^{3}$. Moreover by choosing $G_{i \sigma, j \sigma^{\prime}}\left(z ; z^{\prime}\right) \equiv \delta_{\sigma \sigma^{\prime}} G_{i j}\left(z ; z^{\prime}\right)$ such that $N \equiv-2 \imath \sum_{i} G_{i i}^{<}(t ; t)=2$ for all times, we can ensure that $S_{z}=0$. The results cover the physical parameters $\gamma=1 / 2,1 / 4$ and $\lambda \in[0,2]$ corresponding to the weak- and intermediate-to-strong interactions. The approximate results (H, Gd, GD) are obtained by first solving the imaginary-time Matsubara propagators with an imaginary-time grid, solution method and related parameters identical to the ones used in our previous work in Ref. 1. This leads to multiple solutions characterized by either symmetric or symmetry-broken electron densities and nuclear displacements as shown in Ref. 1. The former kind are the only solutions for a sufficiently weak interaction and are known here as the symmetric solutions, while the latter kind arise for sufficiently strong interactions and are referred to as asymmetric solutions. Here we mention that our approximations do not respect the exact transformation relating the relative and full Hamiltonians of Ref. 1. This is seen as quantitative differences between some equilibirum observables, which are invariant under this transformation in the exact case. presented here and in Ref. 1. In the present work, the real-time electron and phonon propagators are then calculated by time-propagating the Kadanoff-Baym equations, according to an adapted version of the algorithm ${ }^{32}$, using the abovementioned Matsubara propagators either directly or indirectly (see the linear response section) as initial values. The time-grid is uniform with a grid spacing or time-step $\delta_{T}$ such that $t_{\mathrm{kin}} \delta_{T} \in[0.025,0.075]$ extending from zero to the final time $T$ chosen so that $t_{\mathrm{kin}} T=200$. The time-domain propagators are finally Fourier transformed to arrive at their frequency-domain representations. The Fourier transforms are calculated with a high-order quadrature formula and unless otherwise stated by using the Hanning window function ${ }^{70}$.

\section{A. Equilibrium Propagators}

The out-of-equilibrium behavior of a system can be better understood if we first understand the equilibrium properties of this system. These properties are determined by the equilibrium electron and phonon propagators which we have studied in Ref. 1 from the perspective of time-local (e.g. density matrix) and integrated-out (e.g. total energy) observables. Here, we further shed light on the quality of our approximations by investigating the frequency structure of these propagators. In this section, the propagators depend only on the relative time and our convention for evaluating Fourier transforms is that the first time argument is integrated over and the second kept fixed at the initial time.

\section{Electron Propagator}

The electron propagator is directly related to the photoemission i.e. electron removal and inverse photoemission i.e. electron addition spectra. This can be qualitatively seen from its zero-temperature Lehmann representation

$$
G_{i \sigma, j \sigma^{\prime}}^{\gtrless}(\omega)=\mp \imath 2 \pi \sum_{n} f_{n, i \sigma}^{N \gtrless} f_{n, j \sigma^{\prime}}^{N \gtrless}{ }^{*} \delta\left(\omega \mp \Omega_{n}^{N \pm 1}\right),
$$

where $\Omega_{n}^{N \pm 1} \equiv E_{n}^{N \pm 1}-E_{0}^{N}$ is the electron addition/removal energy while $f_{n, i \sigma}^{N>} \equiv\left\langle\Psi_{n}^{N+1}\left|\hat{c}_{i \sigma}^{\dagger}\right| \Psi_{0}^{N}\right\rangle$ and $f_{n, i \sigma}^{N<} \equiv\left\langle\Psi_{n}^{N-1}\left|\hat{c}_{i \sigma}\right| \Psi_{0}^{N}\right\rangle$ are the corresponding amplitudes. Here $\Psi_{n}^{N}$ and $E_{n}^{N}$ denote the $n$th eigenstate and -energy of the $N$ electron system. The Lehmann form is used below to interpret the results shown in Fig. 4 for exact diagonalization (ED) and many-body perturbation theory (H, Gd, GD). The greater and lesser components are related by the particle-hole symmetry $G_{i j}^{>}(\omega)=-(-1)^{i-j} G_{j i}^{<}(-\omega)$ whose fulfillment is discussed below, and therefore we only show results for the lesser component. Let us focus first on the main panels (contour plots) to illustrate the overall frequency structure, and start by examining the exact results. The exact spectra develop as a function of the interaction from the singly peaked, non-interacting spectra described by the function

$$
g_{i j}^{\gtrless}(\omega)=\mp \imath \pi(\mp 1)^{i-j} \delta\left(\omega \mp t_{\text {kin }}\right),
$$

into spectra consisting of multiple peaks whose positions are up to an energy shift given by the energies of the one-electron system. The one-electron energies $E_{n}^{N=1}$ are nearly uniformly separated by the bare phonon frequency for a weak interaction $\lambda \ll 1$. This manifests itself in the exact spectra as emergence of the so-called phonon sideband structure which gains intensity as the initial distribution loses intensity. In the case of a sufficiently strong interactions the lowest energies instead consist of nearly degenerate pairs separated by the bare phonon frequency ${ }^{59,60}$. In this case the one-elecron system can be characterized as polaronic and is, as a first approximation in the limit $\lambda \gg \gamma$, described by

$$
\left|\psi_{k, \pm \sigma}^{\mathrm{LF}}\right\rangle \equiv \frac{1}{\sqrt{2}}\left(\hat{c}_{1 \sigma}^{\dagger} \hat{X} \pm \hat{c}_{2 \sigma}^{\dagger} \hat{X}^{\dagger}\right)|0 ; k\rangle
$$

where $\hat{X} \equiv \exp \left(-\imath g \hat{p} / \omega_{0}\right)$ is a shift operator, and $|0 ; k\rangle$ is an empty electronic state and $k$ th eigenstate of $\hat{a}^{\dagger} \hat{a}^{62}$. In the same limit, we find the two-electron ground state

$$
\left|\Psi_{0}^{\mathrm{LF}}\right\rangle \equiv \frac{1}{\sqrt{2}}\left(\hat{c}_{1 \uparrow}^{\dagger} \hat{c}_{1 \downarrow}^{\dagger} \hat{X}^{2}+\hat{c}_{2 \uparrow}^{\dagger} \hat{c}_{2 \downarrow}^{\dagger} \hat{X}^{\dagger 2}\right)|0 ; 0\rangle .
$$

which has a bipolaronic character. The removal energies and associated amplitudes

$$
\begin{aligned}
\Omega_{k, l \sigma}^{1} & =3 t_{\mathrm{kin}} \lambda / 4+\omega_{0} k, \\
\sum_{\sigma^{\prime}} \sum_{l \in\{ \pm\}}\left|f_{k, l \sigma^{\prime} ; i \sigma}^{2<}\right|^{2} & =\frac{e^{-\lambda / 4 \gamma}}{2 k !}\left(\frac{\lambda}{4 \gamma}\right)^{k} .
\end{aligned}
$$


then show that the spectra consist of peaks separated by the bare phonon frequency with intensities following a Poisson distribution ${ }^{62}$. The exact results shown in Fig. 4 indicate that the initial spectra become denser as interaction is increased such that the two lowest excitations approach one another faster than the third which stays roughly a bare phonon frequency apart, especially for $\gamma=1 / 4$. At the same time spectral weight is redistributed in particular to the third and higher-lying excitations. We interpret this as a precursor of the crossover to a Poissonian disribution which is a signature of a polaronic one-electron and bipolaronic two-electron system. This change is accompanied by an overall shift of the spectra to higher energies which appears smoothly as a function of the interaction, although more rapidly around $\lambda \sim 1$ for the adiabatic ratio $\gamma=1 / 4$. The shift implies that one needs more energy to either add or remove electrons indicating that the two-electron ground state becomes more stable. This is in agreement with the increase in the bipolaron binding energy, see e.g. Ref 1 , and is hence associated with the fact that the two-electron ground state becomes characterizable as bipolaronic. In addition to these changes there is a faint signal around $\omega / t_{\text {kin }} \sim-3$ for $\gamma=1 / 2$ and weak interactions, which is to be understood as the removal energy associated with the anti-bonding state of the one-particle system. This feature is washed out for the lower adiabatic ratio $\gamma=1 / 4$, in contrast to a similar feature of the singleelectron case $^{60}$.

The question is then how well the many-body approximations reproduce the qualitative features of these spectra and thus the associated physics. The Hartree approximation leads to spectra with peaks located at the eigenvalues of the equilibrium Hartree equations. In the case of the symmetric solution, the Hartree potential vanishes, and this approximation just reproduces the noninteracting result $g_{i j}^{\gtrless}(\omega)$ for all values of the interaction thus failing to describe the exact spectra. The asymmetric case however displays a more complicated behavior with the propagators given by

$$
\begin{aligned}
& G_{\mathrm{H}_{a+i j}}^{\gtrless}(\omega)=\imath \pi \lambda^{-1} \delta\left(\omega \mp t_{\mathrm{kin}} \lambda\right), i \neq j, \\
& G_{\mathrm{H}_{a+i} i}^{\gtrless}(\omega)=\mp \imath \pi\left(1 \pm(-1)^{i} \sqrt{1-\lambda^{-2}}\right) \delta\left(\omega \mp t_{\mathrm{kin}} \lambda\right),
\end{aligned}
$$

where $H_{a+}$ denotes the asymmetric solution with a positive density difference $n_{1 \sigma}-n_{2 \sigma}$. The asymmetric spectra emerge at $\lambda=1$, and contain a single peak which moves to higher energy linearly as a function of the interaction. The particle-hole symmetry is broken along with the reflection symmetry, however they are replaced by $G_{\mathrm{H}_{a+} i j}^{\gtrless}(\omega)=G_{\mathrm{H}_{a-j i}}^{\gtrless}(\omega)$ and $G_{\mathrm{H}_{a+i j}}^{\gtrless}(\omega)=$ $-(-1)^{i-j} G_{\mathrm{H}_{a-j i}}^{<}(-\omega)$ where $H_{a-}$ denotes the asymmetric solution with a negative relative density. These relations represent the original symmetries under the interchange of the two degenerate asymmetric solutions. Although the asymmetric solutions lead to spectra which shifts to higher energies as the exact spectra do. they do not show signs of the phonon sideband structure. The Born approximations correct this flaw and show a clear sideband structure. The partially self-consistent Born approximation, in the case of the symmetric solution, however shows that all removal energies behave roughly in a similar fashion, namely they increase monotonously and nearly linearly as a function of the interaction. The spectra do not show signs of a peak corresponding to a removal energy associated with the anti-bonding state of the one-particle system. This feature instead emerges qualitatively correctly in the fully self-consistent approximation. The fully self-consistent approximation also improves the position of the dominant removal energies for weak interactions by showing a stronger increase of the lowest removal energy and a simultaneously decrease in the sideband removal energies. Moreover, on the contrary to the monotonous behavior of the partially selfconsistent approximation, the fully self-consistent approximation shows a signature of a stronger change in the structure of the spectrum for $\lambda=1 / 4$ approximately where the exact spectrum also changes. At this point, the fully self-consistent spectrum however becomes too dense, and does not shift correctly to higher energies. The asymmetric solutions, once they appear for a sufficiently strong interaction, are similar in these approximations and differ from the asymmetric mean-field solution by the fact that there is a related sideband structure.

The changes in the spectra from a non-interacting to a fully interacting case should emerge in a way which respects the two lowest order sum rules for the electron propagator

$$
\begin{aligned}
\mathrm{g}^{(0)} & \equiv \int_{-\infty}^{\infty} \frac{d \omega}{2 \pi \imath} \operatorname{tr} \boldsymbol{G}^{<}(\omega) \\
& =N \\
\mathrm{~g}^{(1)} & \equiv \int_{-\infty}^{\infty} \frac{d \omega}{2 \pi \imath} \omega \operatorname{tr} \boldsymbol{G}^{<}(\omega) \\
& =E_{e}+E_{e p},
\end{aligned}
$$

where the right-hand sides are equilibrium expectation values of the electron number $N$, and electron $E_{e}$ and electron-phonon interaction $E_{e p}$ energies, see Ref. 1. The top panels of Figs. 4 show that both constraints are fulfilled up to a numerical accuracy. The numerical deviations especially for $\gamma=1 / 2$ are due to choice of time discretization and frequency integration. Moreover, we note that all frequency moments have been calculated in the present work from spectra obtained using a rectangular window function. The first moments, which are equal to the mean of the distribution, show that in addition to the asymmetric cases, only the exact and symmetric fully self-consistent spectra move appreciably to higher energies, in particular for $\gamma=1 / 4$.

The left panels of Fig. 4 show the position and intensity of the lowest lying peak labeled with $[E]$, as in Electronic, of the removal spectra. This peak is the most significant 

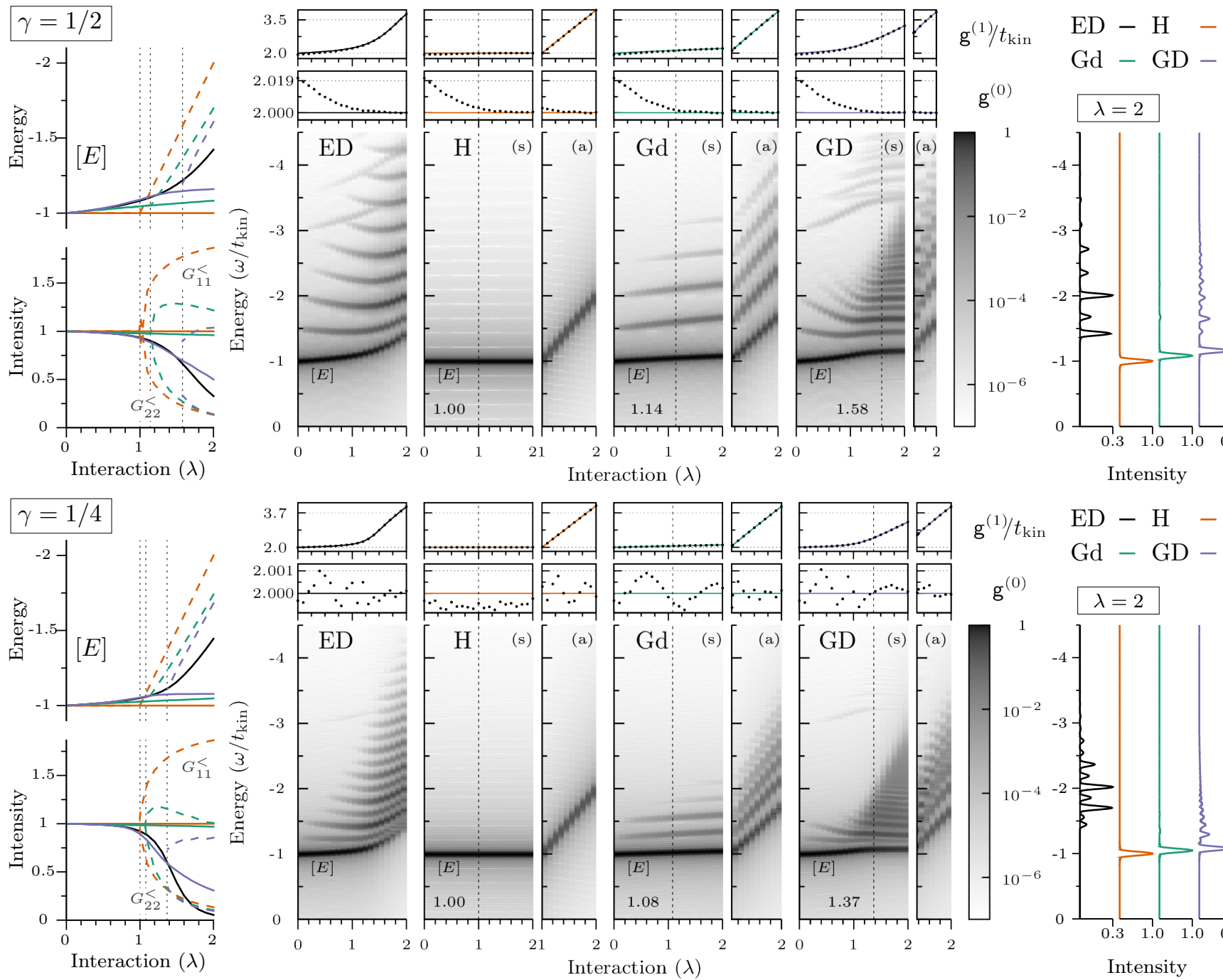

Interaction $(\lambda)$

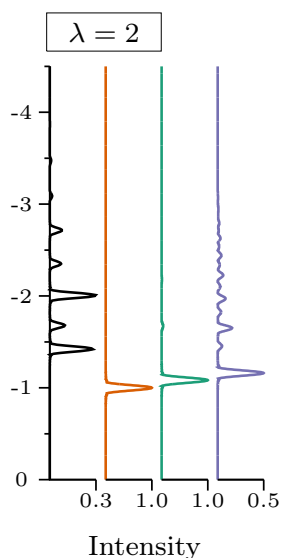

FIG. 4. The exact (ED) and approximate (H, Gd, GD) electron propagator as a function of the interaction $\lambda$ and frequency $\omega$. The top and bottom figures correspond to the adiabatic ratios $\gamma=1 / 2$ and $\gamma=1 / 4$, respectively. The main panels in the middle show $\left|\operatorname{tr} \boldsymbol{G}^{<}(\omega)\right| / T$ for the symmetric (s) solutions and, starting from the critical interaction denoted with a vertical dashed line and its value $\lambda_{C}$, for the asymmetric (a) solutions. The top panels show the zeroth $g^{(0)}$ and first $g^{(1)}$ moments (points) of the spectra and the corresponding expectation values (lines) to illustrate the fulfillment of Eqs. (12). The left panels show the intensities and positions of the lowest energy peak of $2\left|G_{11}^{<}(\omega)\right| / T$ and $2\left|G_{22}^{<}(\omega)\right| / T$, labeled as $[E]$ in the main panels, for the symmetric (solid line) and asymmetric (dashed line) solutions. The right panel show $\left|\operatorname{tr} \boldsymbol{G}^{<}(\omega)\right| / T$ on a linear scale for the symmetric solutions at $\lambda=2$. (color online)

part of the spectra in the regime of weak to intermediate interactions where the many-body approximations are expected to be in qualitative, or even quantitative, agreement with the exact solution. Our results show that, out of the symmetric solutions, the Born approximations are indeed in a good agreement with exact results in the weak coupling regime. The partially self-consistent approximation however deviates considerably already for intermediate interactions $\lambda \sim 1$, while the fully self-consistent approximation gives a reasonably good estimate of both the position and intensity up to borderline strong interactions $\lambda \sim 1.5$. For stronger interactions, both approximations fail to describe the shift of the position, as well as the decrease of the intensity correctly, although the fully self-consistent approximation gets the latter trend considerably better. The exact position and intensity of this peak change more abruptly in the case of $\gamma=1 / 4$, and imply that the sidebands become the most intense part of the exact spectra for the higher interactions considered in this work. The many-body approximations do not show sufficient loss of intensity, and therefore fail to redistribute the spectral weight correctly to the higher energy part. The results for $\lambda=2$ shown in the right panels of Fig. 4 verify this statement and moreover show that the approximate spectra do not bear resemblance to the shape of the exact spectra. Lastly, the asymmetric 
solutions capture the loss of the intensity qualitatively correctly for the site with the lower occupation but in doing so break the reflection symmetry which leads to an increase of the intensity of the site with a higher occupation. This is natural since it becomes favorable to remove electrons from an already almost fully occupied site and vice versa.

To summarize, we found that for the adiabatic ratios considered here the Hartree, and partially and fully selfconsistent Born approximations are in a good agreement with exact results for very weak $\lambda \ll 1$, weak $\lambda<1$, and intermediate $\lambda \sim 1$ interactions, respectively. Moreover, the agreement between exact and approximate results improves when the electronic and phononic energy scale become closer to one another for $\gamma=1 / 2$. These observations are similar to the conclusions of our earlier work in Ref. 1 in which it was further observed that when approaching the anti-adiabatic limit the approximate results start to again deviate from the exact results. In particular, the comparison of the total energies and natural occupation numbers conducted in our previous work supported the view that the fully self-consistent approximation describes the bipolaron crossover partially. The present results show that as the interaction $\lambda$ is increased none of the approximate removal spectra i) move to higher energies as in $\sim 3 t_{\text {kin }} \lambda / 4$ nor ii) develop towards a uniformly $\omega_{0}$-spaced distribution with a Poissonian-like envelope. The results are consistent with our earlier findings as the sum rules are satisfied and e.g. $E_{e}+E_{e p}$ does show a clear significant increase in the fully selfconsistent approximation. As discussed above, points i) and ii) signal a bipolaronic system, and their incorrect description rather suggest the conclusion that none of the approximations describe the bipolaronic crossover even partially. The failure to describe ii) is related to the observation that the intensity of the lowest excitation energy does not decay fast enough as a function of the interaction in the approximate results. This is analogous to the insufficiently fast decaying quasi-particle spectral weight used as an indicator of absence of the bipolaronic metal-insulator transition in the fully self-consistent approximation $^{71}$. Finally, our observation on the relation between the frequency-resolved and integrated-out quantities is similar to those obtained earlier e.g. for the GW approximation in the homogeneous electron gas in which self-consistent total energies were good but the plasmon description inadequate ${ }^{72}$.

\section{Phonon Propagator}

The phonon propagator is an indicator of the properties of the nuclear system, and relates to neutral excitations, as shown by its zero-temperature Lehmann repre- sentation

$$
\begin{aligned}
D_{P Q}^{>}(\omega) & =D_{Q P}^{<}(-\omega) \\
& =-\imath 2 \pi \sum_{n} f_{n, P}^{N} f_{n, Q}^{N}{ }^{*} \delta\left(\omega-\Omega_{n}^{N}\right)
\end{aligned}
$$

where $\Omega_{n}^{N} \equiv E_{n}^{N}-E_{0}^{N}$ is a neutral excitation energy, and $f_{n, P}^{N} \equiv\left\langle\Psi_{n}^{N}\left|\Delta \hat{\phi}_{P}\right| \Psi_{0}^{N}\right\rangle$ the corresponding amplitude. The frequency-domain phonon propagators obtained by means of exact diagonalization (ED) and many-body theory $(\mathrm{H}, \mathrm{Gd}, \mathrm{GD})$ are shown in Fig. 5. Let us first discuss the contour plots which illustrate the overall frequency structure of the spectra. The exact results show that as the interaction is increased the initial, non-interacting spectra described by

$$
\begin{aligned}
& d_{P P}^{>}(\omega)=-\imath 2 \pi \delta\left(\omega-\omega_{0}\right), \\
& d_{P Q}^{>}(\omega)=-2 \pi(P-Q) \delta\left(\omega-\omega_{0}\right), P \neq Q,
\end{aligned}
$$

where $P, Q \in\{1,2\}$ with 1 and 2 referring to the relative displacement and momentum, develop into multipeaked spectra consisting of a low and a high energy scale. The low energy scale consists for a sufficiently strong interaction of a single high intensity peak accompanied by a weaker peak separated approximately by the bare phonon frequency. The high intensity peak which is labeled with $[P]$ referring to Polaronic in the figures, develops continuously from the initial distribution and moves rapidly towards zero energy as a function of the interaction strength. This is true for both adiabatic ratios with the difference that $[P]$ approaches zero more abruptly for $\gamma=1 / 4$. The high energy scale, on the other hand, consists of multiple low intensity peaks above the first electronic excitation energy of the non-interacting system. As the interaction is increased, these excitations move towards higher energies and, although initially gain intensity, become suppressed for a sufficiently strong interaction. These features can be understood from the adiabatic potential energy surfaces defined and analyzed in Ref. 1 and shown here in Fig. 6. This figure shows that the initially quadratic lowest potential energy surface $E_{0}(u)$ becomes more shallow as the interaction is increased which is seen in Fig. 5 as a decreasing phonon frequency. The surface builds up a double-well structure for $\lambda>1$ which manifests itself in the exact results as a nearly degenerate ground and first excited state $[P]$. Moreover, as the barrier between the wells increases, the low energy spectra approach the harmonic spectra of the isolated wells which is seen in the exact results for $\lambda=2$ as a single peak located roughly at the bare phonon frequency. The high-energy spectra, on the other hand, agree with the first excited state surface $E_{1}(u)$ remaining roughly quadratic while the surface separation $E_{1}(u)-E_{0}(u)$ increases. As discussed in Ref. 1, in the adiabatic case $\gamma<1$ the double-well structure is correlated with a splitting of the nuclear ground state probability distribution and the crossover to a bipolaronic state. In this section, we thus identify its spectral 
signature, that is the low energy part consisting of the two peaks, as an indicator of a bipolaronic state.

Let us then focus on the approximate results. The Hartree and partially self-consistent Born approximations approximate the phonon propagator with the noninteracting propagator which does not describe the true behavior of the interacting system discussed above. The question is then how the fully self-consistent approximation, in which the self-energy is a single polarization bubble, fares in this system. In order to answer this, we start with the symmetric solution for which the contour plots of Fig. 5 show that both energy scales of the exact solution are reproduced for the interaction strengths considered. However, in the low energy scale, we only observe $[P]$ and do not find a clear signature of a peak around $\omega / \omega_{0} \sim 1$ for $\lambda \sim 2$ for the propagation times accessed in this work. In the high energy scale, as the interaction is increased the fully self-consistent spectra become denser with non-uniformly separated peaks which do not move as a whole to higher energies. These observations are all in a disagreement with the exact results which show uniformly two bare phonon frequency separated peaks moving to higher energies. This observation is however consistent with the previously discussed properties of the approximate electron propagator for strong interactions. The asymmetric solution, once it is found, is observed to approach the non-interacting result i.e. the lowest frequency approaches the bare phonon frequency and higher lying structure looses intensity as the interaction is increased. This is expected since there is no room for particle-hole excitations in the symmetry-broken system, and thus the polarization bubble should tend to zero when the interaction is increased. As in the electronic case, also these spectra should fulfill sum rules given in terms of the zeroth and first moments by

$$
\begin{aligned}
\mathrm{d}^{(0)} & \equiv-\int_{-\infty}^{\infty} \frac{d \omega}{2 \pi \imath} \operatorname{tr} \boldsymbol{D}^{>}(\omega) \\
& =\operatorname{tr} \boldsymbol{\Lambda} \\
\mathrm{d}^{(1)} & \equiv-\int_{-\infty}^{\infty} \frac{d \omega}{2 \pi \imath} \omega \operatorname{tr}\left(\boldsymbol{\alpha} \boldsymbol{D}^{>}(\omega)\right) \\
& =2 E_{p Q}+E_{e p Q}+\operatorname{tr}\left(\boldsymbol{\alpha} \boldsymbol{\Omega}^{M}\right),
\end{aligned}
$$

where $\boldsymbol{\Lambda} \equiv \imath \boldsymbol{D}^{M}\left(0^{+}\right)$, and $E_{p Q}$ and $E_{e p Q}$ are the quantum contributions to the phonon and electron-phonon interaction energies defined in Ref. 1. The top panels of Fig. 5 show that these sum rules are approximately obeyed, and therefore an important consistency relation is satisfied. Here it is noteworthy that although there is a clear change in the phonon energy, see the zeroth frequency moment, in the exact and fully selfconsistent solutions, only the former displays a clear kink at $\lambda \sim 1.3-1.5$ in the first frequency moment. Finally, the top panels of Fig. 5 highlight the lowest excitation energy labeled with $[P]$ which is the dominant part of
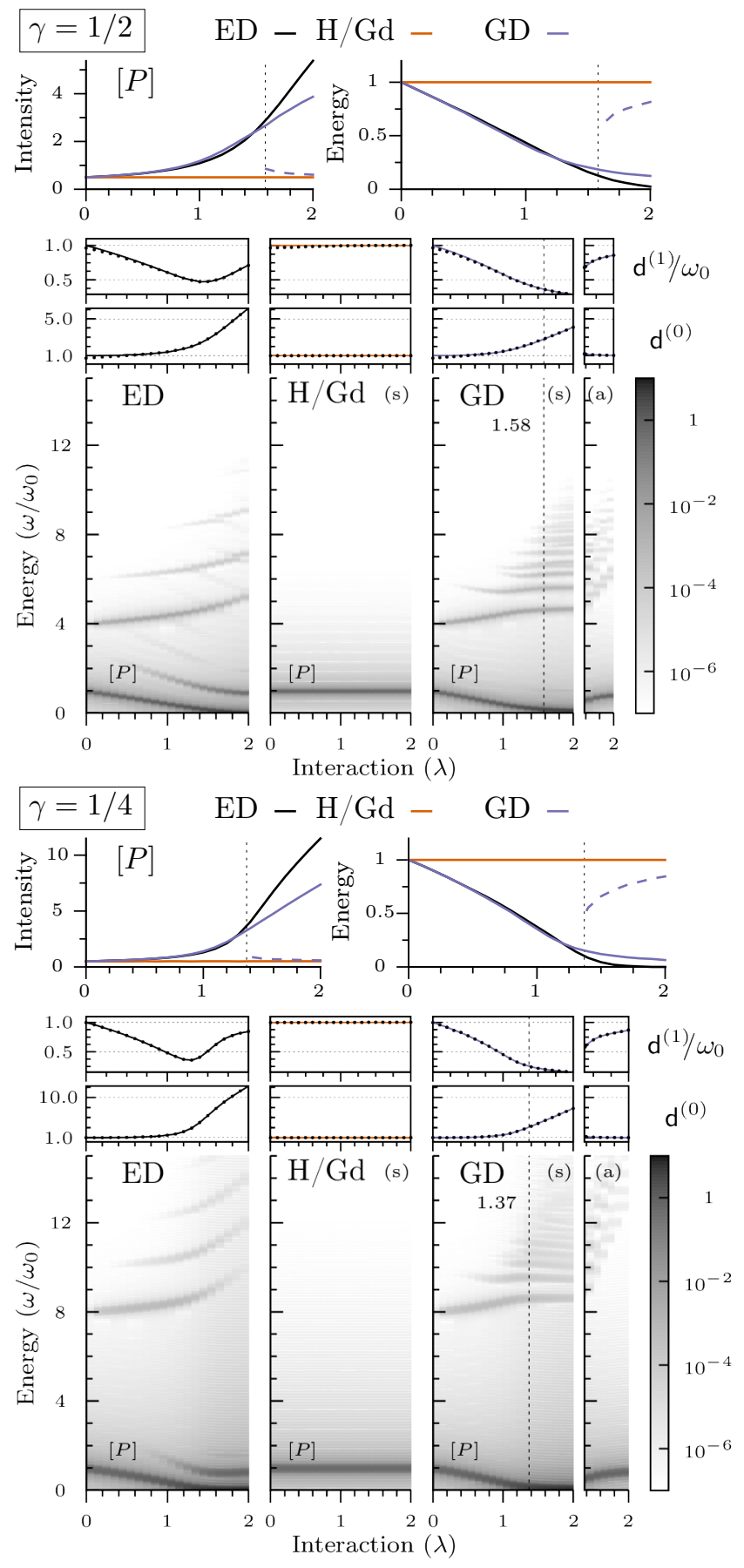

FIG. 5. The exact (ED) and approximate (H, Gd, GD) phonon propagator as a function of the interaction $\lambda$ and frequency $\omega$. The top and bottom figures relate to the adiabatic ratio $\gamma=1 / 2$ and $\gamma=1 / 4$, respectively. The contour plots show $\left|D_{11}^{>}(\omega)\right| / T$ on logarithmic scale for the symmetric (s) solutions and, starting from the critical interaction marked with a vertical dashed line and its value $\lambda_{C}$, for the asymmetric (a) solutions. The middle panels show the zeroth $\mathrm{d}^{(0)}$ and first $d^{(1)}$ moments (points) of the spectra and the corresponding expectation values (lines) to illustrate fulfillment of Eqs. (13). The top panels show the intensities and positions of the lowest energy peak of $\left|D_{11}^{>}(\omega)\right| / T$ for the symmetric (solid line) and asymmetric (dashed line) solutions labeled as $[P]$ in the contour plots. (color online) 
the spectra. The exact results show that this peak approaches zero energy, but never actually reaches it, and gains intensity as a function of the interaction. This is in contrast to the non-interacting propagator in which this sole feature remains at the bare phonon frequency. The self-consistent Born approximation however captures both effects reasonably accurately up to $\lambda \sim 1.5$ and gives a qualitatively similar trend even beyond it for the interactions considered here.

To summarize, we found that the non-interacting propagator used in the Hartree and partially self-consistent Born approximations is an adequate approximation for the interacting phonon propagator only for very weak $\lambda \ll 1$ interactions. The fully self-consistent Born approximation, on the other hand, captures the dominant low energy peak well up to borderline strong $\lambda \sim 1.5$ interactions and is therefore in a good agreement with the exact results in this range of interactions. It however does not describe the second low energy excitation at $\omega_{0}$, and therefore reproduces qualitatively only one of the signatures of a bipolaronic state observable in the phonon propagator. Finally, we remark that the absence of a peak at the bare phonon frequency for strong interactions is a likely factor for the observed too dense frequency structure of the electron propagator.

\section{B. Linear Response Functions}

The dynamics of a system of electrons and nuclei is in many cases dominantly determined by a linear response function provided that the system is perturbed sufficiently weakly. Many spectroscopic methods essentially rely on measuring these functions which makes them important for understanding experiments. Here we investigate in particular the first order density-density response function of our model system.

\section{Method}

Let us begin by explaining how we in practice calculate linear response functions in our time-dependent formalism. This is a prerequisite for understanding when it is reasonable to do linear response by time-propagation. The density-density response function is calculated by perturbing the system with the time-dependent potential

$$
\hat{V}(t)=\delta(t) \sum_{i \sigma} v_{i} \hat{n}_{i \sigma}
$$

where $v_{i}$ is the magnitude of the perturbation, and $\delta$ is the Dirac delta function. Then we record the resulting spin-summed density

$$
n_{i}(t)=-2 \imath G_{i i}^{<}(t ; t),
$$

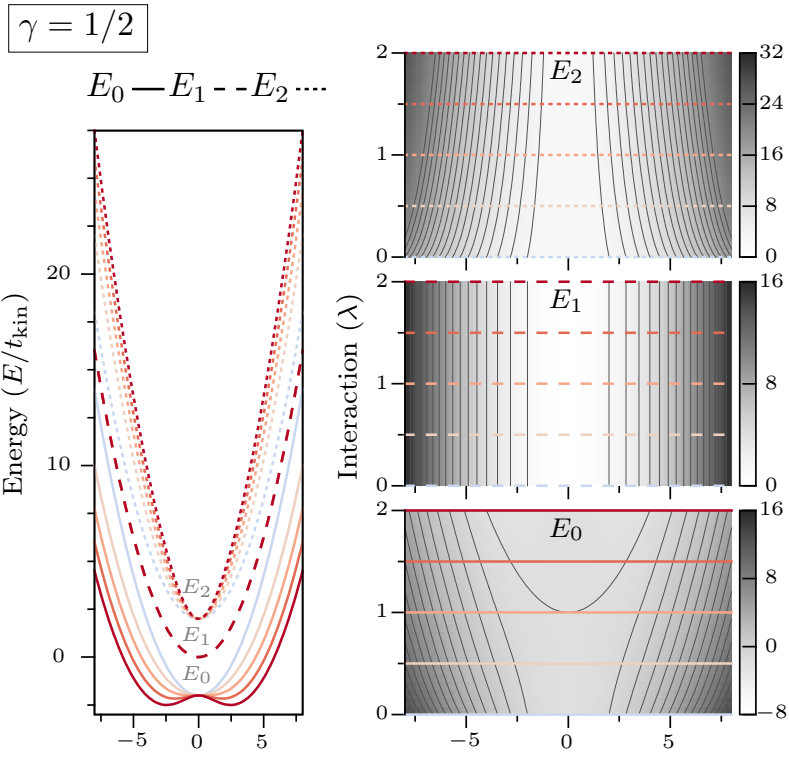

Relative Displacement $(u)$

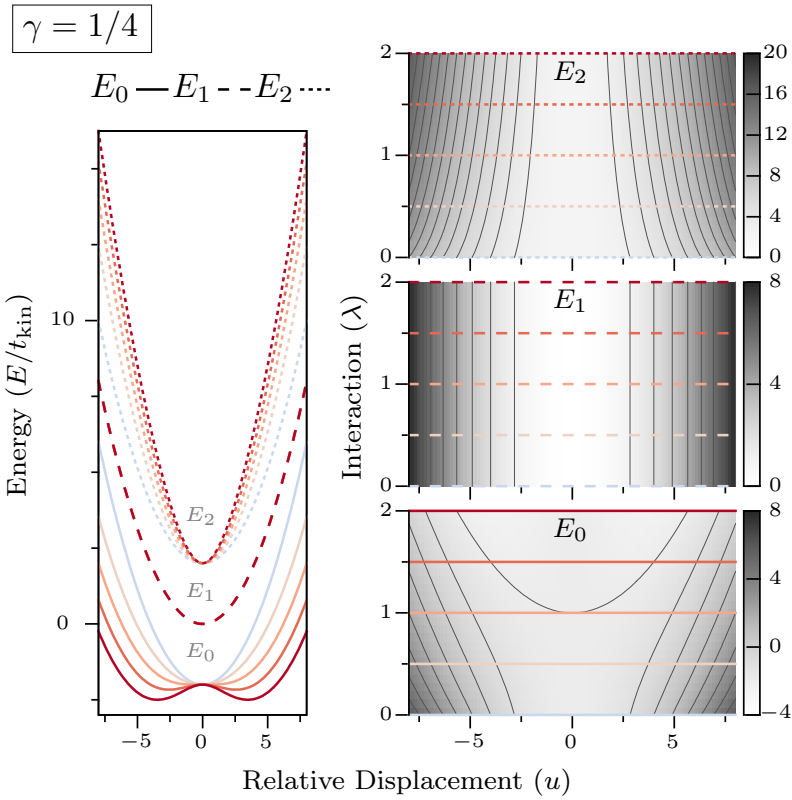

FIG. 6. The adiabatic potential energy surfaces $E_{0}(u), E_{1}(u)$ and $E_{2}(u)$ for the three singlet eigenstates of the electronic clamped nuclei Hamiltonian as a function of the interaction $\lambda$ and relative displacement $u$, see Ref. 1 for details. The top and bottom figures correspond to the adiabatic ratios $\gamma=1 / 2$ and $\gamma=1 / 4$, respectively. The left panels contain $\lambda=0,0.5,1.0,1.5,2.0$ cross-sections of the potential energy surfaces shown as contour plots in the right panels. (color online)

which in the linear response regime satisfies

$$
\begin{aligned}
\delta n_{i}(t) & \equiv n_{i}(t)-n_{i}^{(0)}(t) \\
& =\sum_{j} \chi_{i j}^{R}(t) v_{j}+\mathcal{O}\left(v^{2}\right),
\end{aligned}
$$


where $v$ is the norm of a vector with $v_{i}$ as its components, $n_{i}^{(0)}(t)$ is the density of the unperturbed system, and $\chi_{i j}^{R}(t) \equiv \sum_{\sigma \sigma^{\prime}} \chi_{i \sigma, j \sigma^{\prime}}^{R}(t)$ the retarded component of the first order density-density response function. The response function is then given by

$$
\left.\chi_{i j}^{R}(t) \equiv \frac{\partial n_{i}(t)}{\partial v_{j}}\right|_{v=0},
$$

which we in practice evaluate by using the difference quotient $\left(n_{i}(t)-n_{i}^{(0)}(t)\right) / v_{j}$ with $v_{j}$ sufficiently small and $v_{k}=0$ for $k \neq j$. Lastly, it is important to understand that applying the delta function potential amounts to choosing a new initial state after which the timeevolution is induced by the unperturbed Hamiltonian. In exact diagonalization, this is achieved by preparing the new initial state

$$
\left|\tilde{\Psi}_{0}^{N}\right\rangle=e^{-\imath \sum_{i \sigma} v_{i} \hat{n}_{i \sigma}}\left|\Psi_{0}^{N}\right\rangle
$$

where $\left|\Psi_{0}^{N}\right\rangle$ is the $N$ electron ground state, and which is subsequently propagated in the absence of the perturbation. In the Kadanoff-Baym equations, on the other hand, the same is achieved by choosing the new initial electron propagators

$$
\begin{aligned}
& G_{i j}^{\gtrless}(0 ; 0)=e^{-\imath\left(v_{i}-v_{j}\right)} G_{i j}^{\mathrm{M}}\left(0^{ \pm}\right), \\
& G_{i j}^{\dagger}(0 ; \tau)=e^{-\imath v_{i}} G_{i j}^{M}(-\tau),
\end{aligned}
$$

where $G_{i j}^{\mathrm{M}}(\tau)$ is the solution to the equilibrium Dyson equation. The electron and phonon propagators are then obtained by time-propagating the unperturbed KadanoffBaym equations.

\section{Stability}

The method described above is expected to work if the perturbation expansion of Eq. (15) is valid for the time scales of interest. It can however be that a possibly finite time-scale in which the expansion is good cannot be extended to cover the entire time-scale of interest. This can signal e.g. an unbounded linear response function. In the following, we show that this is the case for the Hartree approximation, and subsequently investigate whether or not the Born approximations show a similar behavior.

The Hartree Eqs. (10) are a closed set of ordinary differential equations for the phonon field expectation value $\phi_{P}(t)$ and the electron propagator $G_{i j}^{<}(t ; t)$. In the twosite, two-electron Holstein model these equations can be rewritten as

$$
\begin{aligned}
\dot{n} & =4 t_{\mathrm{kin}} \Gamma_{2}, \\
\dot{\Gamma}_{1} & =-2 g u \Gamma_{2}, \\
\dot{\Gamma}_{2} & =-t_{\mathrm{kin}} n+2 g u \Gamma_{1}, \\
\dot{u} & =\omega_{0} p, \\
\dot{p} & =-\omega_{0} u+2 g n,
\end{aligned}
$$

where we have suppressed the time arguments and the overhead dot denotes the time-derivative. Moreover, $n \equiv n_{1 \sigma}-n_{2 \sigma}, u \equiv\left(u_{1}-u_{2}\right) / \sqrt{2}$, and $p \equiv\left(p_{1}-p_{2}\right) / \sqrt{2}$ are the relative spin density, displacement, and momentum, while $\Gamma_{1}$ and $\Gamma_{2}$ are the real and imaginary parts of the density matrix element $\gamma_{12} \equiv-\imath G_{12}^{<}$, respectively. As shown explicitly in App. B, the density-density response function is the solution to the corresponding linearized equations of motion. If the linearization is performed with respect to an equilibrium solution which is a stable fixed-point, in the sense of Lyapunov ${ }^{73-75}$, of the original equations then the eigenvalues of the resulting Jacobian matrix have non-positive real parts ${ }^{73}$. Moreover, if there are no repeated zero eigenvalues, then the zero solution of the linearized system is stable and furthermore any solution is bounded ${ }^{75}$. In particular, the density response function is then bounded, that is $\exists M>0$ independent of $t$ such that $\left|\chi_{i j}^{R}(t)\right| \leq M$ for all $t \geq 0$. In order understand when this is the case, we investigate below the stability of the fixed-points of the Hartree equations. The fixed-points whose stability is to be studied are just the symmetric

$$
\begin{aligned}
n_{s} & =0, \\
\Gamma_{s, 2} & =0, \\
u_{s} & =0, \\
p_{s} & =0,
\end{aligned}
$$

and asymmetric

$$
\begin{aligned}
n_{a \pm} & = \pm \sqrt{1-\lambda^{-2}}, \\
\Gamma_{a \pm, 2} & =0 \\
u_{a \pm} & =2 g n_{a \pm} / \omega_{0}, \\
p_{a \pm} & =0
\end{aligned}
$$

solutions of the equilibrium Hartree equations of Eqs. (A2) derived in Ref. 1. These equations are subject to two constants of motion as both the eigenvalues of the reduced density matrix, which are either one or zero, and the total energy are conserved and give the constraints

$$
\begin{aligned}
1 & =n^{2}+4\left(\Gamma_{1}^{2}+\Gamma_{2}^{2}\right), \\
E & =\frac{\omega_{0}}{2}\left(p^{2}+u^{2}-1\right)-4 t_{\mathrm{kin}} \Gamma_{1}-2 g n u,
\end{aligned}
$$

respectively. Then by following ${ }^{76,77}$, and motivated by the first constraint, we introduce the coordinates

$$
\begin{aligned}
z & =2 \Gamma_{1}, \\
n & =\sqrt{1-z^{2}} \cos (\theta), \\
\Gamma_{2} & =\sqrt{1-z^{2}} \sin (\theta) / 2,
\end{aligned}
$$

which represent cross-sections of the unit sphere with a 
plane. The transformed equations of motion

$$
\begin{aligned}
& \dot{\theta}=-2 t_{\text {kin }}+2 g \frac{u z \cos (\theta)}{\sqrt{1-z^{2}}}, \\
& \dot{z}=-2 g u \sqrt{1-z^{2}} \sin (\theta), \\
& \dot{u}=\omega_{0} p, \\
& \dot{p}=-\omega_{0} u+2 g \sqrt{1-z^{2}} \cos (\theta),
\end{aligned}
$$

and the total energy

$$
E=\frac{\omega_{0}}{2}\left(p^{2}+u^{2}-1\right)-2 t_{\mathrm{kin}} z-2 g u \sqrt{1-z^{2}} \cos (\theta),
$$

then correspond to a Hamiltonian system with canonical conjugate variables $(\theta, z)$ and $(u, p)$. The canonical transformation

$$
\begin{aligned}
q_{1} & =-\sqrt{2(1-z)} \sin (\theta), \\
p_{1} & =\sqrt{2(1-z)} \cos (\theta), \\
q_{2} & =-p \\
p_{2} & =u
\end{aligned}
$$

transforms this system into two non-linearly coupled oscillators described by

$$
\begin{aligned}
E & =\frac{\omega_{0}}{2}\left(p_{2}^{2}+q_{2}^{2}\right)+t_{\mathrm{kin}}\left(p_{1}^{2}+q_{1}^{2}\right) \\
& -2 g p_{2} p_{1} \sqrt{1-\left(p_{1}^{2}+q_{1}^{2}\right) / 4}
\end{aligned}
$$

where we dropped a constant energy shift. This system of equations is a special case of the Hamiltonian system studied in ${ }^{76}$ which arises from the semi-classical equations of the Dicke model ${ }^{76,78}$. Let us then denote $x_{1} \equiv q_{1}$, $x_{2} \equiv q_{2}, x_{3} \equiv p_{1}$, and $x_{4} \equiv p_{2}$. The fixed points of this system are just related by coordinate transforms to the fixed points of Eqs. (21). At the symmetric fixed-point, we find the Hessian matrix

$$
\nabla \nabla E_{s} \equiv\left(\begin{array}{cccc}
2 t_{\text {kin }} & 0 & 0 & 0 \\
0 & \omega_{0} & 0 & 0 \\
0 & 0 & 2 t_{\text {kin }} & -2 g \\
0 & 0 & -2 g & \omega_{0}
\end{array}\right)
$$

which is positive-definite for $\lambda<1$ and indefinite for $\lambda>1$, while at the asymmetric fixed-points, the Hessian matrix

$$
\nabla \nabla E_{a \pm} \equiv\left(\begin{array}{cccc}
t_{\mathrm{kin}}(1+\lambda) & 0 & 0 & 0 \\
0 & \omega_{0} & 0 & 0 \\
0 & 0 & \frac{4 t_{\mathrm{kin}} \lambda}{1+\lambda^{-1}} & -\frac{2 \sqrt{2} g \lambda^{-1}}{\sqrt{1+\lambda^{-1}}} \\
0 & 0 & -\frac{2 \sqrt{2} g \lambda^{-1}}{\sqrt{1+\lambda^{-1}}} & \omega_{0}
\end{array}\right)
$$

is positive-definite for $\lambda>1$. The symmetric equilibrium and asymmetric equilibria are then due to the LagrangeDirichlet theorem ${ }^{79,80}$ stable for $\lambda<1$ and $\lambda>1$, respectively. Moreover, since $\operatorname{det}\left(\nabla \nabla E_{s}\right)<0$ for $\lambda>1$ also $\operatorname{det}\left(\boldsymbol{J} \nabla \nabla E_{s}\right)<0$, where $\boldsymbol{J}$ is the standard symplectic matrix ${ }^{79}$, and therefore the Jacobian matrix of the linearized Hamilton's equations has an eigenvalue with a negative real-part. This implies that there also exists an eigenvalue with a positive real part which means that the equations are linearly and nonlinearly unstable $^{75}$. The symmetric equilibrium is therefore unstable for $\lambda>1$. The zero solution losing its stability while two new stable equilibria arise is a standard bifurcation known as the supercritical pitchfork bifurcation ${ }^{81}$. The stability together with the fact that the Hessian matrices do not have zero eigenvalues for $\lambda \neq 1$ implies that the response functions obtained for $\lambda<1$ and $\lambda>1$ using the symmetric equilibrium and asymmetric equilibria are bounded functions. The linear instability of the symmetric solution for $\lambda>1$ leads, on the other hand, to an unbounded response function as shown in App. B.

This answers the question when it is in this context appropriate to do linear response properties at the meanfield level, but does not resolve this issue for the correlated approximations. In this case, one cannot recast the equations as a set of ordinary differential equations, but instead must consider the full two-time integrodifferential equations which have non-linear integral kernels. As we are not aware of stability theory for such dynamical systems and it would go beyond the scope of the present work, we only resort to a working measure which is in the spirit of the stability of the equilibrium solutions. The working measure chosen here is a practical one: we introduce the norm

$$
\|\delta n\|_{\infty} \equiv \max _{t \in[0, T]}|n(t)-n(0)|,
$$

compare it to the magnitude of the perturbation $v$, and if they remain in the same order of magnitude, we suggest that the equilibrium is stable. We emphasize that this measure is not equal to the stability even in the case of ordinary differential equations, but does give a practical estimate whether or not a linear response calculation makes sense for the time scales accessed in this work.

Having said this, Fig. 7 shows this norm for exact diagonalization (ED) and many-body theory (H, Gd, GD). The results are obtained either by starting from a symmetric, or asymmetric equilibrium solution. The exact results stay always in the same magnitude as the perturbing potential $v=10^{-3}$ which is expected since the timedependent Schrödinger equation is linear. The other extreme is the symmetric mean-field solution for which the norm suddenly, although continuously as a function of the interaction, approaches one when the interaction exceeds the corresponding critical value $\lambda=1$. At the same time, this norm remains in the same order of magnitude as the perturbation for the asymmetric mean-field solution. This is consistent with the stability analysis presented above illustrates that our working measure agrees in this case with Lyapunov stability. These cases give us some confidence in looking at the symmetric and asymmetric solutions of the Born approximations. The results for the asymmetric cases of these approximations indicate that they behave qualitatively similar to the asymmetric equilibria of the mean-field suggesting that they are 

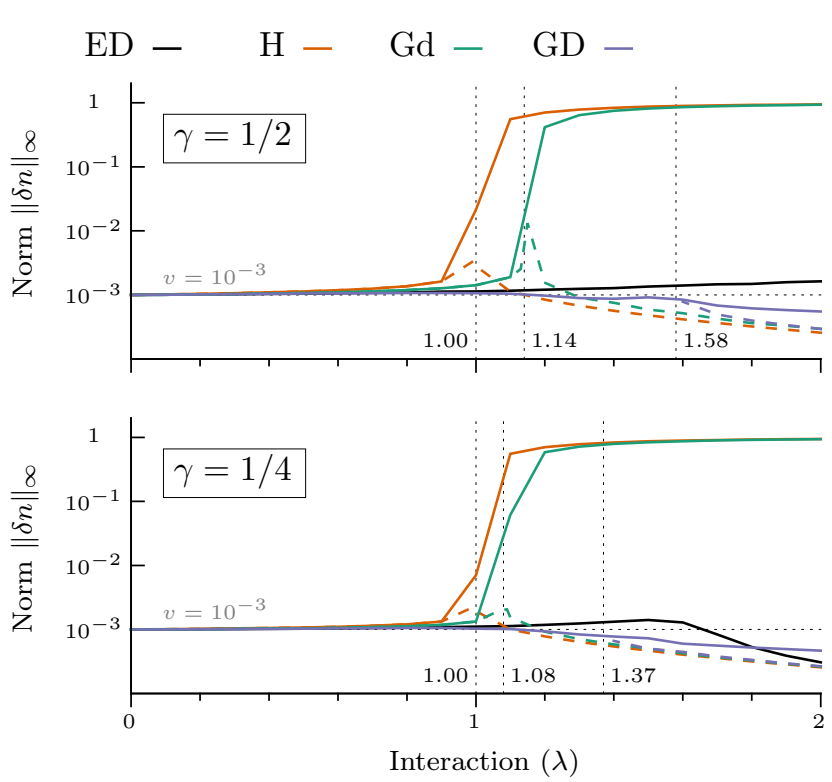

FIG. 7. The norm $\|\delta n\|_{\infty}$ of Eq. (23) as a function of the interaction $\lambda$ for the perturbation of Eq. (14) with $v_{1}=10^{-3}, v_{2}=0$. The top and bottom panels correspond to the adiabatic ratios $\gamma=1 / 2$ and $\gamma=1 / 4$, respectively. The solid and dashed lines correspond to the symmetric and asymmetric solutions, respectively. The vertical dashed lines and the associated values $\lambda_{C}$ denote the critical interactions which are ordered according to to H, Gd, and GD from left to right. (color online)

stable. This qualitative agreement remains true for the symmetric equilibria of the partially self-consistent case in which the norms approach one when passing the critical value of interaction. The norm of the symmetric solution of the fully self-consistent approximation however remains in the same order of magnitude as the perturbation. We note that the declining norm for $\gamma=1 / 4$ and higher interactions is in the exact case related to the fact that the propagation length is shorter than the period of the lowest excitation of the system.

These observations together with the interpretation given to our working measure of stability then suggest that the partially self-consistent approximation has the same qualitative stability properties as the mean-field. On the other hand, both equilibrium solutions of the fully self-consistent Born approximation are observed to be stable in the sense of our working measure. This means that, on the contrary to the Hartree and partially selfconsistent Born approximation, it is possible to do linear response with respect to the symmetric equilibrium of the fully self-consistent Born approximation for the time-scales addressed here.

\section{Bethe-Salpeter equation}

The Bethe-Salpeter equation is the standard approach to calculate linear response functions in many-body perturbation theory ${ }^{40}$. Here, we discuss the connection between the many-body approximations used in this frequency-domain approach and in the time-dependent approach applied in the present work. We start by noting that the density response function of Eq. (16) is the retarded $\chi_{i j}^{R}(t)=\sum_{\sigma \sigma^{\prime}} \chi_{i \sigma i \sigma, j \sigma^{\prime} j \sigma^{\prime}}^{R}(t ; 0)$ component of the generalized, contour-time response function

$\chi_{i j, k l}\left(z ; z^{\prime}\right) \equiv \frac{1}{\imath \mathcal{Z}} \operatorname{Tr}\left[\mathcal{T}\left\{e^{-\imath \int_{C} d \bar{z} \hat{H}(\bar{z})} \Delta \hat{\gamma}_{i j}(z) \Delta \hat{\gamma}_{k l}\left(z^{\prime}\right)\right\}\right]$,

where $\hat{\gamma}_{i j} \equiv \hat{c}_{j}^{\dagger} \hat{c}_{i}$ is the one-body reduced density matrix operator and $\Delta \hat{\gamma}_{i j}$ the corresponding fluctuation operator. Note that we switched here to collective indices containing both spatial and spin degrees of freedom. In the standard approach, the generalized response function satisfies the equation

$$
\begin{aligned}
\chi_{i j, k l}\left(z ; z^{\prime}\right) & =P_{i j, k l}\left(z ; z^{\prime}\right)+\sum_{P Q} \sum_{r s t u} \int_{C} d \bar{z} d \underline{z} P_{i j, r s}(z ; \bar{z}) \\
& \times M_{s r}^{P}(\bar{z}) d_{P Q}(\bar{z} ; \underline{z}) M_{t u}^{Q}(\underline{z}) \chi_{u t, k l}\left(\underline{z} ; z^{\prime}\right), \\
P_{i j, k l}\left(z ; z^{\prime}\right) & =-\imath \sum_{p q} \int_{C} d \bar{z} d \underline{z} G_{i p}(z ; \bar{z}) \\
& \times G_{q j}(\underline{z} ; z) \Gamma_{p q ; k l}\left(\bar{z}, \underline{z} ; z^{\prime}\right),
\end{aligned}
$$

where $P_{i j, k l}\left(z ; z^{\prime}\right)$ is the irreducible polarizability defined in terms of the irreducible vertex function $\Gamma_{i j, k l}\left(z, z^{\prime} ; z^{\prime \prime}\right)$. These equations are valid for any many-body approximation which includes the mean-field, Hartree term while beyond mean-field effects are incorporated into the irreducible vertex function. This function satisfies the BetheSalpeter equation ${ }^{2}$

$$
\begin{aligned}
\Gamma_{i j, k l}\left(z, z^{\prime} ; z^{\prime \prime}\right) & =\delta_{i l} \delta_{j k} \delta\left(z, z^{\prime}\right) \delta\left(z^{\prime}, z^{\prime \prime}\right) \\
& +\sum_{p q r s} \int_{C} d \bar{z} d \bar{z}^{\prime} d \underline{z} d \underline{z}^{\prime} K_{i q, p j}\left(z, \bar{z} ; \bar{z}^{\prime}, z^{\prime}\right) \\
& \times G_{p r}\left(\bar{z}^{\prime} ; \underline{z}\right) G_{s q}\left(\underline{z}^{\prime} ; \bar{z}\right) \Gamma_{r s, k l}\left(\underline{z}, \underline{z}^{\prime} ; z^{\prime \prime}\right),
\end{aligned}
$$

where the four-point integral kernel is defined as

$$
K_{i j, k l}\left(z, z^{\prime} ; \bar{z}, \bar{z}^{\prime}\right) \equiv \frac{\delta \Sigma_{\mathrm{xc}, i l}\left(z ; \bar{z}^{\prime}\right)}{\delta G_{k j}\left(\bar{z} ; z^{\prime}\right)},
$$

with the subscript xc denoting the exchange-correlation self-energy. The diagrammatic form of this equation is shown in the top panel of Fig. 8. It has been shown ${ }^{37}$ that a density response function obtained by time-propagation of the Kadanoff-Baym equations with a self-energy $\Sigma$ is equivalent to a solution of Eqs.(25) with the vertex satisfying the Bethe-Salpeter equation with a four-point kernel of Eq. (26). Thus by calculating the response function 


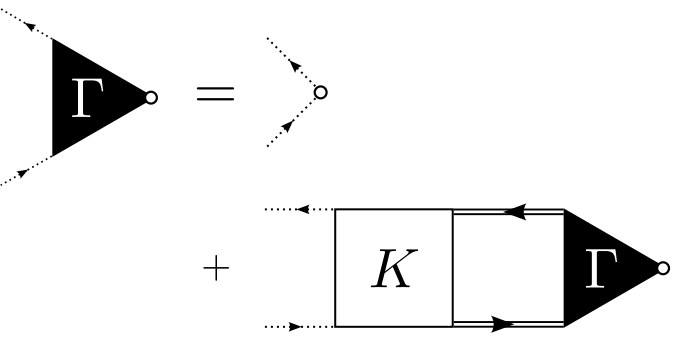

$K_{\mathrm{H}}=\emptyset$
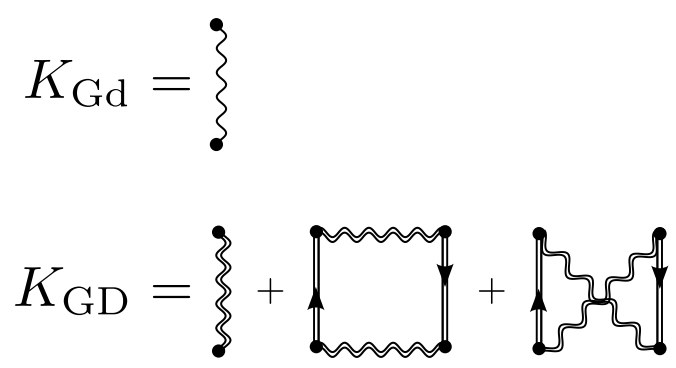

FIG. 8. The Bethe-Salpeter equation for the irreducible vertex function (top) and functional forms of the four-point kernels (bottom) in the Hartree (H), and the partially (Gd) and fully (GD) self-consistent Born approximations. A line with an arrow indicates a dressed electron propagator, while single and two-fold wiggly lines represent bare and dressed phonon propagators, respectively. An open circle represents a connection for a phonon propagator and a closed circle (kernel) or a dashed line (BSE) a connection for an electron propagator.

via time-propagation using Eqs. (15) and (16) we arrive by current standards at a high-level solution of the BetheSalpeter equation.

Figure. 8 shows diagrammatically the approximate four-point kernels related to the self-energy approximations used in the present work. In the Hartree approximation, the irreducible vertex function is the bare vertex, and the response function is hence the sum of all bubble diagrams. The partially self-consistent Born approximation leads to a vertex function which consists of all the ladder diagrams and to the bubbles-and-ladders series for the response function. In addition to such terms, the fully self-consistent Born approximation contains also two second order kernel diagrams in terms of the phonon propagators. These higher-order terms are not routinely considered in the zero-frequency ${ }^{54,55}$ nor fully frequencydependent $\operatorname{cases}^{40}$. Lastly, we note that despite of the sophistication of these approximations there are in general no guarantees of their superiority over the conventional approximations. It has also been shown, that similar approximations in the purely electronic case, can lead to undesired features like non-positivity ${ }^{82}$.

\section{Density-Density Response Function}

We have presented a stability analysis in order to understand when it makes sense to calculate linear response properties in the context of the present work. Additionally, we have discussed the diagrammatic meaning of the response function obtained in this manner. Here we focus on the numerical results, that is analyzing the densitydensity response function obtained by time-propagation of the Kadanoff-Baym equations. In order to carry-out this analysis, we start by considering the exact response function which has the frequency-domain Lehmann representation

$$
\chi_{i j}^{R}(\omega)=\sum_{n}\left(\frac{h_{n i}^{N} h_{n j}^{N}}{\omega-\Omega_{n}^{N}+\imath \eta}-\frac{h_{n i}^{N} h_{n j}^{N}}{\omega+\Omega_{n}^{N}+\imath \eta}\right),
$$

where $\Omega_{n}^{N} \equiv E_{n}^{N}-E_{0}^{N}$ is a neutral excitation energy, and $h_{n, i}^{N} \equiv\left\langle\Psi_{0}^{N}\left|\sum_{\sigma} \hat{n}_{i \sigma}\right| \Psi_{n}^{N}\right\rangle$ the corresponding real-valued oscillator strength. This form is used to analyze the exact (ED) density response function which is shown together with the approximate $(\mathrm{H}, \mathrm{Gd}, \mathrm{GD})$ response functions in Fig. 9. The main panels contain contour plots which illustrate the overall structure of the spectra. The exact results show that the non-interacting response function

$$
\chi_{0 ; i j}^{R}(\omega) \equiv \frac{(-1)^{i-j} / 2}{\omega-2 t_{\mathrm{kin}}+\imath \eta}-\frac{(-1)^{i-j} / 2}{\omega+2 t_{\mathrm{kin}}+\imath \eta},
$$

which consists of a single peak for positive frequencies, develops as a function of the interaction to a function comprising multiple excitation energies. In the case of weak $\lambda<1$ interactions, these excitations can be reasonably well identified as phonon sidebands i.e. as multiphonon excitations either from the non-interacting electronic ground or first, singly-excited, excited state. The sidebands corresponding to either electronic state are separated roughly by two bare phonon frequencies for weak interactions. Moreover, there is an extremely faint peak located at $\omega / t_{\text {kin }} \sim 4.5$ for $\gamma=1 / 2$ coinciding energetically with the non-interacting doubly-excited electronic state plus a single phonon. As the interaction is increased, the structure associated initially with the singly-excited electronic state labeled with $[E]$ in the figures moves as a whole to higher energies. At the same time, the peaks related initially to the electronic ground state approach a bare phonon frequency separated distribution with the lowest excitation labeled with $[P]$ in the figures approaching zero energy and gaining intensity. These spectral features can be understood from the adiabatic potential energy surfaces of Fig. 6 as discussed in Sec. IV A 2. In the following, we instead focus on the dominant low energy peak $[P]$ with the aim to identify it as a signature of a bipolaronic system. We start by writing the exact time-dependent density as

$$
n_{i}(t)=\int_{-\infty}^{\infty} d u\left(2 P_{i i}(u ; t)+P_{12}(u ; t)\right),
$$



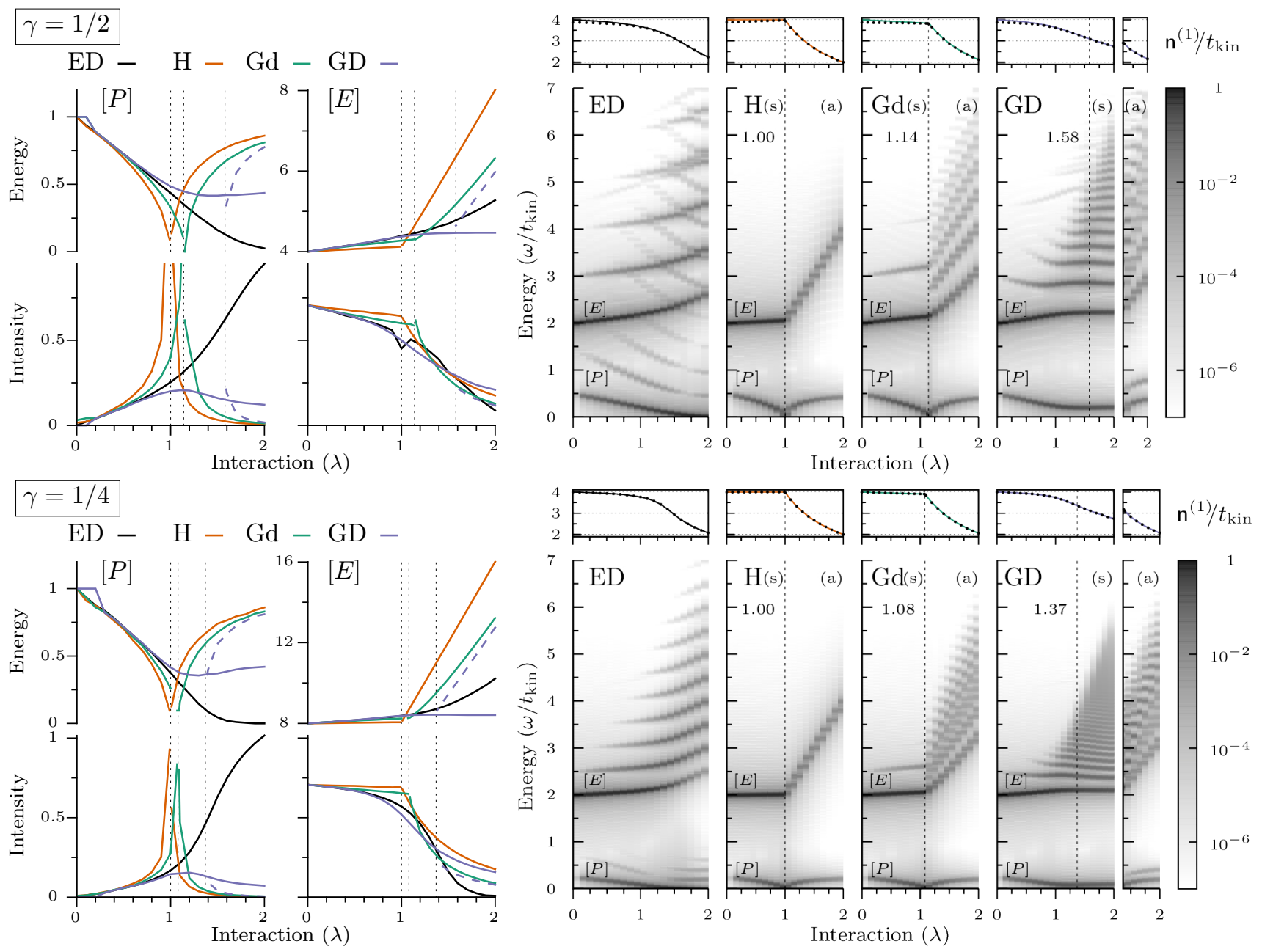

FIG. 9. The exact (ED) and approximate (H, Gd, GD) retarded density-density response function as a function of the interaction $\lambda$ and frequency $\omega$. The top and bottom panels correspond to the adiabatic ratios $\gamma=1 / 2$ and $\gamma=1 / 4$, respectively. The contour plots on the right show $\left|\operatorname{tr} \chi^{R}(\omega)\right| / T$ for the stable symmetric (s) or asymmetric (a) solutions. The small top panels show the first $n^{(1)}$ moment (points) and the corresponding expectation value (lines) to illustrate fulfillment of Eq. (28). The left panels show the intensities and positions of the two peaks of $\left|\operatorname{tr} \chi^{R}(\omega)\right| / T$ labeled with $[P]$ and $[E]$ in the contour plots. The stable solutions of $\mathrm{H}$ and Gd are denoted with a solid line irrespective of their symmetry. The stable symmetric and asymmetric solutions of GD are denoted with a solid and dashed line, respectively. (color online)

where $P_{i j}(u ; t)$ defined by

$$
\begin{aligned}
P_{i i}(u ; t) & \equiv\left|\left\langle i \uparrow, i \downarrow ; u \mid \tilde{\Psi}_{0}^{N=2}(t)\right\rangle\right|^{2}, \\
P_{12}(u ; t) & \equiv \sum_{\substack{\sigma \sigma^{\prime} \\
\sigma \neq \sigma^{\prime}}}\left|\left\langle 1 \sigma, 2 \sigma^{\prime} ; u \mid \tilde{\Psi}_{0}^{N=2}(t)\right\rangle\right|^{2},
\end{aligned}
$$

is the time-dependent joint probability to find the electrons at sites $i$ and $j$ and nuclei at the relative coordinate $u$ at time $t$. Here we use the notation $\left|\tilde{\Psi}_{0}^{N=2}(t)\right\rangle \equiv$ $\exp \left(-\imath \hat{H}^{M} t\right)\left|\tilde{\Psi}_{0}^{N=2}\right\rangle$ with $\left|\tilde{\Psi}_{0}^{N=2}\right\rangle$ defined in Eq. (17) and $\left|i \sigma, j \sigma^{\prime} ; u\right\rangle \equiv \hat{c}_{i \sigma}^{\dagger} \hat{c}_{j \sigma^{\prime}}^{\dagger}|0\rangle_{e}|u\rangle$ where $|0\rangle_{e}$ is the electronic vacuum and $|u\rangle$ is an eigenstate of $\hat{u}$. The exact density response function can be then according to Eq. (16) written as

$$
\chi_{i j}^{R}(t)=2 \int_{-\infty}^{\infty} d u \varrho_{i i, j}^{R}(u ; t)
$$

where

$$
\left.\varrho_{i k, j}^{R}(u ; t) \equiv \frac{\partial P_{i k}(u ; t)}{\partial v_{j}}\right|_{v=0},
$$

is a response function describing how the ground state joint probability $P_{i j}(u ; 0)$ changes as a function of time due to a weak perturbation. The response function $\varrho_{12, j}(u ; t)$ does not contribute to the density response function since it is an odd function under the interchange $u \leftrightarrow-u$ as follows from the full inversion symmetry of the model. In Ref. 1, we use the ground state joint probabilities as ingredients of a working definition of a 
dominantly bipolaronic ground state. The probabilities $P_{i j}(u ; 0)$ shown in the top panels of Fig. 10 illustrate the fact that as the interaction increases one is most likely to find the system in a state in which both electrons occupy the same site with an accompanying nuclear displacement. In particular, at $\lambda=2.0$ for $\gamma=1 / 2$ and $\lambda=1.7$ for $\gamma=1 / 4$, the ground state of the system has according to Ref. 1 crossed over to a dominantly bipolaronic state. Next, we illustrate how these distributions behave in the linear response regime by showing the time-average

$$
\left\langle\varrho_{i k, j}\right\rangle(u) \equiv \frac{1}{T} \int_{0}^{T} d t\left|\varrho_{i k, j}(u ; t)\right|,
$$

as a function of the interaction and displacement in the left contour plots of Fig. 10. The final time $T$ is chosen here so that $T / t_{\text {kin }}^{-1} \approx 470$ and $T / t_{\text {kin }}^{-1} \approx 9360$ for $\gamma=1 / 2$ and $\gamma=1 / 4$, respectively. The results indicate that i) $\varrho_{11,1}(u, t)$ and $\varrho_{22,1}(u, t)$ are on average larger than $\varrho_{12,1}(u, t)$, and that for a sufficiently strong interactions the latter become suppressed while the former gain magnitude. The maxima $\max _{u, t \in[0, T]}\left|\varrho_{i k, j}(u ; t)\right|$ shown in the insets underneath the averages further support these statements. Moreover, we observe that when the ground state distributions become spatially polarized as the interaction is increased, also the response functions follow the same trend. Thus we find that ii) the spatial shapes of the initial distributions remain qualitatively invariant in the linear response regime as a function of time. In order to illustrate the temporal behavior of the dominant response functions $\varrho_{i i, 1}(u ; t)$, we show them in the right panels of Fig. 10 for the initially bipolaronic systems at $\lambda=2.0$ and $\lambda=1.7$ for $\gamma=1 / 2$ and $\gamma=1 / 4$, respectively. Firstly, these results agree with the conclusion ii) on the spatial structure, and secondly, they show that iii) probability density is redistributed between $\varrho_{11,1}(u ; t)$ and $\varrho_{22,1}(u ; t)$ mainly on a time-scale given by the energy scale of $[P]$, while the energy scale given by $[E]$ is seen as superimposed small amplitude oscillations. The points i), ii) and ii) combined allow us to conclude that, in agreement with the working definition of Ref. 1, the system is in a dominantly bipolaronic state at each instant of time. Moreover, we understand the oscillation of the probability density between $\varrho_{11,1}(u ; t)$ and $\varrho_{22,1}(u ; t)$ to represent the motion of a bipolaron appearing according to iii) on a time scale set by $[P]$. Finally, this is seen in the density response function according to Eq. (27) as the emergence of the dominant low energy excitation $[P]$.

As we have described the exact density response function, we are prepared to investigate how the many-body approximations describe it in order to understand their limitations. Let us begin with the mean-field, Hartree approximation in which the density response function can be obtained analytically as the solution to the linearized Hartree equations as shown in App. B. This response function is given by

$$
\chi_{\mathrm{H}_{n} ; i j}^{R}(\omega) \equiv \sum_{k \in\{ \pm\}}\left(\frac{(-1)^{i-j} \chi_{k}^{n} / 2}{\omega-\omega_{k}^{n}+\imath \eta}-\frac{(-1)^{i-j} \chi_{k}^{n} / 2}{\omega+\omega_{k}^{n}+\imath \eta}\right),
$$
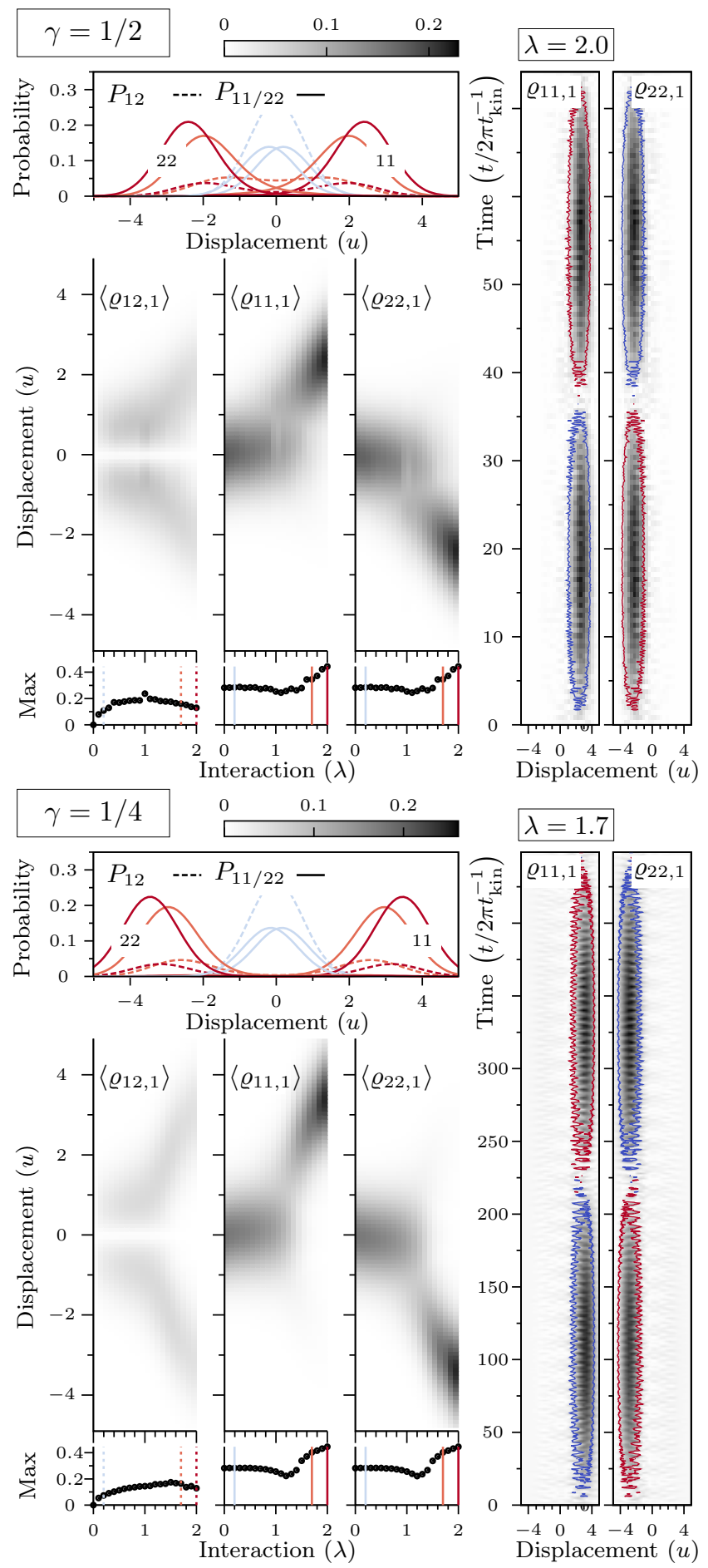

FIG. 10. The exact (ED) joint-probabilities $P_{i j}(u ; 0)$ and response functions $\varrho_{i k, j}(u ; t)$ shown for the adiabatic ratios $\gamma=$ $1 / 2$ and $\gamma=1 / 4$ in the top and bottom figures, respectively. In the top panels, we show $P_{i j}(u ; 0)$ as a function of the displacement $u$ for the interactions $\lambda=0.2,1.7,2.0$ whose color code is shown in the bottom panels. The middle panels contain $\left\langle\varrho_{i j, 1}\right\rangle$ as a function of the displacement and interaction, and the bottom panels display $\max u, t \in[0, T]\left|\varrho_{i j, 1}(u ; t)\right|$ as a function of the interaction. In right panels, we show $\varrho_{i i, 1}(u ; t)$ as a function of the displacement and time $t$ for the interactions $\lambda=2.0,1.7$ with regions closed by red (blue) contour lines being positive (negative) values. (color online) 
where

$$
\chi_{ \pm}^{n} \equiv \frac{2 t_{\mathrm{kin}}\left(\omega_{0}^{2}-\omega_{ \pm}^{n 2}\right)^{2}}{\omega_{ \pm}^{n}\left[\left(\omega_{0}^{2}-\omega_{ \pm}^{n 2}\right)^{2}+4 \lambda \omega_{0}^{2} t_{\mathrm{kin}}^{2}\right]}
$$

are the oscillator strengths and

$$
\begin{aligned}
& \omega_{ \pm}^{s} \equiv \sqrt{\frac{\omega_{0}^{2}+4 t_{\text {kin }}^{2}}{2}\left(1 \pm \sqrt{1+\frac{16 \omega_{0}^{2} t_{\text {kin }}^{2}(\lambda-1)}{\left(\omega_{0}^{2}+4 t_{\text {kin }}^{2}\right)^{2}}}\right)}, \\
& \omega_{ \pm}^{a} \equiv \sqrt{\frac{\omega_{0}^{2}+4 t_{\text {kin }}^{2} \lambda^{2}}{2}\left(1 \pm \sqrt{1-\frac{16 \omega_{0}^{2} t_{\text {kin }}^{2}\left(\lambda^{2}-1\right)}{\left(\omega_{0}^{2}+4 t_{\text {kin }}^{2} \lambda^{2}\right)^{2}}}\right)},
\end{aligned}
$$

are the frequencies for the symmetric $(s) \lambda<1$ and asymmetric $(a) \lambda>1$ solutions, respectively. The Hartree response functions shown in the subpanels $\mathrm{H}$ of Fig. 9 thus consists of two contributions: the high $[E]\left(\chi_{+}^{n}, \omega_{+}^{n}\right)$ and low $[P]\left(\chi_{-}^{n}, \omega_{-}^{n}\right)$ energy peaks related, for weak interactions, to the non-interacting electronic excited and ground states plus zero and one phonon, respectively. Firstly, we observe that as the interaction is increased, the initial distribution given by $[E]$ remains nearly invariant up to the critical interaction $\lambda=1$ beyond which its energy increases linearly as a function of the interaction. Secondly, the low energy peak $[P]$, which has no intensity in the non-interacting $\lambda=0$ case, gains intensity and approaches the zero energy as the interaction is increased. Moreover, when it reaches the zero energy at the critical interaction $\lambda=1$, its intensity given by the oscillator strength $\chi_{-}^{n}$ diverges. As explained in Sec.IV B 2, by increasing the interaction beyond this point, we make the symmetric equilibrium solution unstable, and therefore we change the initial state to one of the asymmetric solutions. This leads again to a well-defined first order response with the intensity of the low energy peak becoming finite and its frequency approaching the bare phonon frequency as the interaction is increased. We understand these results in terms of the adiabatic potential energy surfaces so that the mean-field approximation captures the lowest adiabatic potential energy surface of Fig. 6 becoming more shallow which leads to the lowest excitation approaching the zero energy. This agrees with the observation that, as the Hessian matrices of Sec. IV B 2 indicate, there is a direction in the energy landscape in the neighborhood of the symmetric equilibrium solution such that along it, as $\lambda \rightarrow 1$, the approximately harmonic energy surface becomes more shallow. Moreover, at $\lambda=1$, this harmonic surface becomes completely flat, and as the linearized equations describe only this local neighborhood, it appears as if exciting the system costs no energy which manifests itself as the divergence of the zero-frequency component of the response function. At this point, the lowest adiabatic potential energy surface forms the double-well structure which has for $\lambda=1$ also a locally flat energy landscape at $u=0$ where its second derivative vanishes. Lastly, one can show that the Hartree ground state energy function is equivalent to the lowest adiabatic potential energy surface $E_{0}(u)$ by enforcing in Eq. (22) that $2 g n=u$ and that $\Gamma_{1}$ satisfies the equilibrium Hartree equations derived in Ref. 1. This suggests that the Hartree approximation captures the formation of the double-well potential but needs to fall into one of the two minima in order to minimize the energy. By doing so, it sees again a nearly harmonic surface, which appears in Fig. 9 as the lowest excitation becoming finite and approaching the bare phonon frequency.

Let us then discuss the density response functions obtained for the stable equilibrium solutions of the partially (Gd) and fully (GD) self-consistent Born approximations shown in Fig. 9. The partially self-consistent results shown in the subpanel Gd of Fig. 9 indicate that the main qualitative difference to the Hartree approximation is that there is a sideband structure related to the excitation $[E]$. The sidebands are separated roughly by two bare phonon frequencies in agreement with the exact results for weak interactions, but do not move to higher energies as a function of the interaction as clearly as the exact spectra does. Moreover, when $\lambda$ exceeds $\lambda_{C}$, the ground state becomes asymmetric and new symmetry-forbidden excitations emerge in-between the original sidebands. In the low energy scale, we instead do not observe new qualitative differences to the mean-field solution, in particular we still find that at $\lambda_{C}$, the low energy peak $[P]$ reaches the zero energy with its intesity diverging. The symmetric solution of the fully self-consistent Born approximation does, however, show a qualitative difference as shown in the subpanel GD (s) of Fig. 9. In the low energy scale, we observe that as the interaction is increased, the low energy peak $[P]$ moves initially towards the zero energy but, in contrast to the mean-field and partially self-consistent results, does not reach it for the parameters considered in this work. This is in an agreement with the exact solution in which, however, the lowest excitation becomes increasingly close to the ground state, while in the fully self-consistent approximation, we observe that it approaches a finite non-zero value. In the high energy scale, we on the other hand observe that as the interaction is increased, the peaks above $[E]$ become non-uniformly spaced and too dense in comparison to the exact solution. These shortcomings, as well as the fact that the spectra do not move appreciably to higher energies as a function of the interaction, are similar to what we observed for the equilibrium electron propagators in Sec. IV A 1. Finally, the asymmetric solutions shown in the subpanels GD (a) are similar to the partially self-consistent solutions for $\lambda>\lambda_{C}$ except for the additional excitation at $\omega / \omega_{0} \sim 2$. The low energy sidebands seen in the exact solution are then likely merely symmetry-forbidden in the symmetric solution of the fully self-consistent approximation. As the last remark on the overall structure, as shown in the top insets of Fig. 9, the development of these spectra as a function 
of the interaction is consistent with the f-sum rule

$$
\begin{aligned}
\mathrm{n}^{(1)} & \equiv-\int_{-\infty}^{\infty} \frac{d \omega}{\pi \imath} \omega \operatorname{tr} \chi^{R}(\omega) \\
& =-2\left(E_{e}-E_{e}^{\mathrm{loc}}\right)-2\left(E_{e p}-E_{e p}^{\mathrm{loc}}\right)
\end{aligned}
$$

which relates the first moment of the density-density response function to the electron $\left(E_{e}, E_{e}^{\mathrm{loc}}\right)$ and electronphonon interaction energies $\left(E_{e p}, E_{e p}^{\mathrm{loc}}\right)$, where superscript loc refers to the site-diagonal part of the corresponding energy. The fact that also the many-body approximations satisfy this sum rule is proven for the purely electronic case in in $^{38,83}$.

Next, we focus on the two most significant features of the density response function for the weak and intermediate interactions. These are the intensity and position of $[P]$ and $[E]$ shown in the left panels of Fig. 9. In the exact case, as the interaction is increased, $[E]$ loses magnitude and moves towards higher energies while $[P]$ gains intensity and approaches the zero energy. The former is dominant up to borderline strong $\lambda \sim 1.5$ interactions although the latter is appreciable already for $\lambda \sim 1$, and its impact to the response properties is emphasized by its different energy scale. In the Hartree approximation, we observe that, as a function of the interaction, $[E]$ is nearly invariant for $\lambda<1$ and that $[P]$ behaves in a divergent manner as desribed above. The mean-field approximation therefore agrees with the exact solution only for very weak interactions $\lambda \ll 1$. The partially self-consistent Born results are qualitatively similar to the mean-field results. On a quantitative level, it reproduces the exact intensity and position of $[E]$ better but deviates considerably for intermediate $\lambda \sim 1$ interactions. This together with the observed divergence of $[P]$ implies that it can be said to agree well with exact resutls only for weak $\lambda<1$ interactions. In both approximations, we find that the intensities of $[E]$ and $[P]$ decrease rapidly as a function of the interaction once $\lambda$ exceeds $\lambda_{C}$. The density response to a weak parturbation is thus suppressed for a sufficiently strong interaction which is consistent with the localized nature of the asymmetric equilibrium solutions discussed in Ref. 1. Moreover, we find that also the asymmetric solution of the fully self-consistent Born approximation behaves qualitatively in this manner for the interactions it has been found. Lastly, the symmetric solution of the fully self-consistent Born approximation reproduces the exact positions and intensities of $[E]$ and $[P]$ well up to intermediate interactions $\lambda \sim 1$ with the intensity of $[E]$ being good also for stronger interactions. In this approximation, the low energy excitation $[P]$ does not reach the zero energy nor does it diverge as a function of the interaction, but its position and intensity do not still agree even qualitatively with the exact solution for strong $\lambda>1$ interactions.

To summarize, we have found similarly as in Sec. IV A 1 that the Hartree, and partially and fully selfconsistent Born approximations are in a good agreement with the exact results for very weak $\lambda \ll 1$, and weak $\lambda<1$ and up to intermediate $\lambda \sim 1$ interactions. In particular, we have shown that the exact density response function has, for sufficiently strong $\lambda>1$ interactions, a dominant low energy excitation which none of the approximation describe qualitatively correctly. Moreover, we have related this excitation to the response of a bipolaron to a weak perturbation by analyzing it using the time-dependent joint probabilities. Instead of describing the low energy excitation, the Hartree and partially selfconsistent Born approximations give rise to a divergence of the response function at the critical point $\lambda_{C}$. This has been explained by relating it to the formation of the double-well structure in the lowest adiabatic potential energy surface which we have also associated with the bipolaronic crossover in Ref. 1. Finally, we have shown that the divergence can be prevented by dressing the phonon propagator self-consistently at the level of the fully selfconsistent Born approximation.

\section{Phonon Propagator Revisited}

The density response function which we calculated and discussed above describes electron density fluctuations which in turn couple to nuclear density fluctuations described by the phonon propagator. This is formally shown by the Dyson equation of Eq. (4b) which can be written as

$$
\begin{aligned}
\boldsymbol{D}\left(z ; z^{\prime}\right) & =\boldsymbol{d}\left(z ; z^{\prime}\right) \\
& +\int_{C} d \bar{z} d \bar{z}^{\prime} \boldsymbol{d}(z ; \bar{z}) \boldsymbol{\Pi}_{r}\left(\bar{z} ; \bar{z}^{\prime}\right) \boldsymbol{d}\left(\bar{z}^{\prime} ; z^{\prime}\right) \\
\Pi_{r, P Q}\left(z ; z^{\prime}\right) & \equiv \sum_{i j k l} M_{j i}^{P}(z) \chi_{i j, k l}\left(z ; z^{\prime}\right) M_{l k}^{Q}\left(z^{\prime}\right)
\end{aligned}
$$

where the reducible self-energy $\Pi_{r}$ is determined by the generalized response function of Eq. (24). This means that a density response function obtained by timepropagation can be also used to obtain a new phonon propagator. Here we use this relation to identify some phonon self-energies and discuss whether or not they lead to better nuclear properties than the fully self-consistent Born approximation (GD). In order to do this, due to computational reasons instead of using the equilibrium frequency-domain version of Eq. 29, we perturb the system with the instantaneous force

$$
\hat{F}_{P}(t)=\delta(t) F_{P}
$$

where $F_{P}$ is the magnitude of the perturbation. We then record the resulting phonon field expectation value which in the linear response regime satisfies

$$
\begin{aligned}
\delta \phi_{P}(t) & \equiv \phi_{P}(t)-\phi_{P}^{(0)}(t) \\
& =\sum_{Q} D_{P Q}^{R}(t) F_{Q}+\mathcal{O}\left(F^{2}\right)
\end{aligned}
$$


where $\phi_{P}^{(0)}(t)$ is the expectation value of the unperturbed system, and $D_{P Q}^{R}(t)$ is the retarded phonon propagator. In the Kadanoff-Baym equations this perturbation amounts to choosing

$$
\phi_{P}(0)=\phi_{P}^{M}-\imath \sum_{Q} \alpha_{P Q} F_{Q},
$$

where $\phi_{P}^{M}$ is the equilibrium expectation value, as the new initial condition and subsequently solving the equations of motion in the absence of this perturbation. The phonon propagator is then given by

$$
\left.D_{P Q}^{R}(t) \equiv \frac{\partial \phi_{P}(t)}{\partial F_{Q}}\right|_{F=0} .
$$

which we in practice evaluate by using the difference quotient $\left(\phi_{P}(t)-\phi_{P}^{(0)}(t)\right) / F_{Q}$ with sufficiently small $F_{Q}$ and $F_{R}=0$ for $R \neq Q$. It can be shown that this propagator satisfies Eq. (29) and its irreducible version of Eq. (4b) with the irreducible self-energy

$$
\Pi_{P Q}\left(z ; z^{\prime}\right)=\sum_{i j k l} M_{j i}^{P}(z) P_{i j, k l}\left(z ; z^{\prime}\right) M_{l k}^{Q}\left(z^{\prime}\right),
$$

where $P$ is the irreducible polarizability of Eq. (25b). The phonon propagators obtained by time-propagation are thus related to irreducible self-energy functionals whose lowest-order diagrammatic expansions are shown in Fig. 11. The phonon propagator obtained in this manner in the Hartree $(\mathrm{H})$, and partially $(\mathrm{Gd})$ and fully (GD) self-consistent Born approximations are respectively given by

$$
\begin{aligned}
\Pi_{\mathrm{td}-\mathrm{H}}\left(z ; z^{\prime}\right) & =\Pi_{\mathrm{B}}\left[G_{\mathrm{H}}, d\right]\left(z ; z^{\prime}\right), \\
\Pi_{\mathrm{td}-\mathrm{Gd}}\left(z ; z^{\prime}\right) & =\Pi_{\mathrm{BL}}\left[G_{\mathrm{Gd}}, d\right]\left(z ; z^{\prime}\right), \\
\Pi_{\mathrm{td}-\mathrm{GD}}\left(z ; z^{\prime}\right) & =\Pi_{\mathrm{BLX}}\left[G_{\mathrm{GD}}, D_{\mathrm{GD}}\right]\left(z ; z^{\prime}\right),
\end{aligned}
$$

where we have introduced the prefix 'td-' referring ot time-dependent to distinguish these self-energies from their original counterparts. This shows explicitly that these approximations are not self-consistent i.e. the propagator satisfying the Dyson equation is not the same as the ones in the self-energy diagrams.

The results can be anticipated by noting that the noninteracting phonon propagator is a function peaked at the bare phonon frequencies. The reducible frequencydomain Dyson equation then suggests that the phonon propagator satisfying it has a similar frequency content as the density response function with weight redistributed around the bare phonon frequencies. This already gives a picture how good are the phonon propagators obtained from the density response functions of Sec. IV B 4. However, let us try to make this picture more quantitative. Figure 12 shows the Fourier transforms of the retarded phonon propagators obtained by time-propagation for the many-body approximations (td-H, td-Gd, td-GD). The exact (ED) and fully self-consistent Born (GD) equilibrium propagators are also shown for reference. We
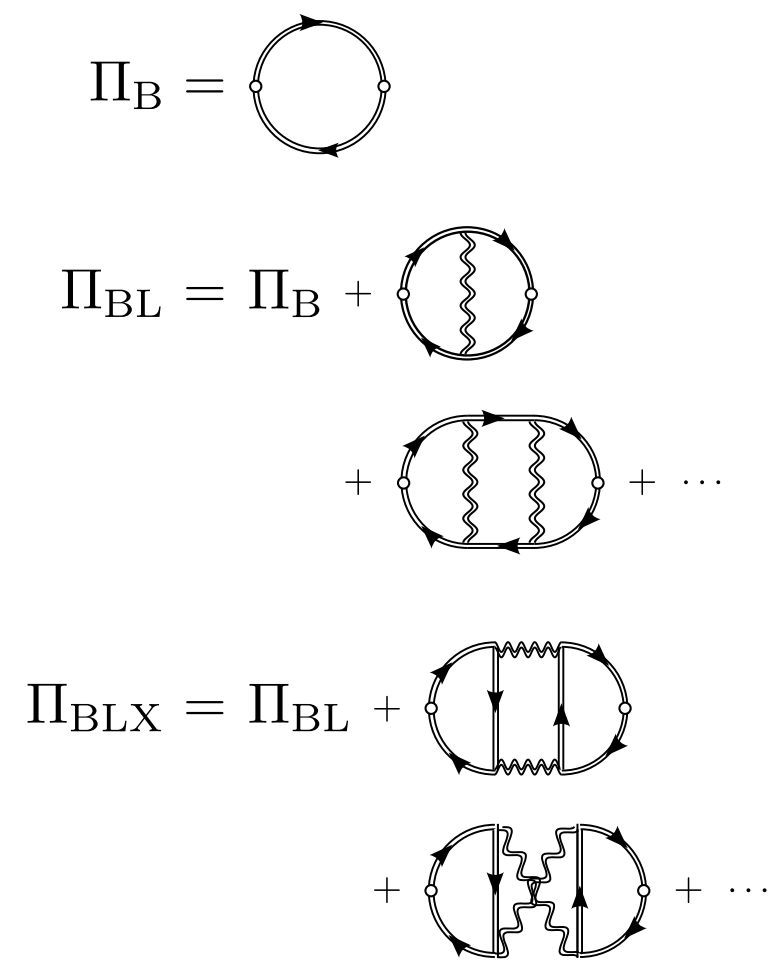

FIG. 11. The phonon self-energies, or their functional forms, corresponding to the phonon propagators obtained by timepropagation. The Hartree $(\mathrm{H})$, and partially $(\mathrm{Gd})$ and fully (GD) self-consistent Born approximations relate to the bubble $(B)$, and bubble-ladders (BL) and bubble-ladders-exchange (BLX) self-energy functionals, respectively. A line with an arrow indicates a dressed electron propagator, while single and two-fold wiggly lines represent bare and dressed phonon propagators, respectively. An open circle represents a connection for a phonon propagator.

have discussed the reference results and their physical content in Sec. IV A 2 so here we focus directly to comparing the different approximations. The contour plots show that none of the new approximations improve the qualitative description of the low energy peak $[P]$ which dominates the spectra. Moreover, only td-Gd with a symmetry-broken ground state for $\lambda>\lambda_{C}$ produces a sideband structure for this excitation. We also observe that td-GD has a slightly larger sideband separation for the electronic excitation when compared to the fully selfconsistent Born (GD) spectra. Here we remark that the numerical results for td-H agree with the analytical results and discussion on the phonon vacuum instability presented in Ref. 1. Lastly, we show the position and intensity of $[P]$ relative to its exact position and intensity in the top panels of Fig. 12 for weak interactions $\lambda<0.5$. The results highlight, as expected in a perturbative regime, that $\mathrm{td}-\mathrm{H}$ deviates the most and $\mathrm{td}-\mathrm{GD}$ the least from the exact result. Furthermore td-Gd and GD give similar results in this regime with the former being slightly better as it includes all the diagrams up to fourth order in the electron-phonon interaction. 
Overall due to the poor description of the lowest excitation, the td-H, td-Gd, and td-GD approximations are only valid in the regime of weak interactions $\lambda<1$ in which td-Gd and td-GD improve on GD. The qualitative behavior for larger interactions $\lambda \sim 1$ shows that although one can obtain sophisticated many-body selfenergies by means of time-propagation, they do not necessarily improve the description of the physics. In particular, we find that infinite summation schemes for selfenergy diagrams, which include vertex corrections, lead to deterioration of the spectral properties of the equilibrium propagator evaluated with a single dressed polarization bubble for intermediate to high interactions.

\section{v. CONCLUSIONS AND OUTLOOK}

We have introduced a method based on timedependent many-body perturbation theory aimed at studying interacting electrons and phonons. The manybody approximations used here are the Hartree $(\mathrm{H})$, and the partially (Gd) and fully (GD) self-consistent Born approximations. The method has been applied to investigate both the non-neutral and neutral excitation spectra of a two-site, two-electron Holstein model. We have presented results for the frequency-domain groundstate electron and phonon propagators, as well as for the density-density and displacement-displacement linear response functions. The results have been compared with numerically exact results obtained by exact diagonalization (ED) in order to assess their quality and relate a physical picture to the behavior of the many-body approximations.

In Ref. 1, we found that the approximations studied here support multiple, concurrently co-existing solutions some of which exhibit a broken reflection symmetry. The asymmetric solutions were found once the electronphonon interaction $\lambda$ reached a critical value $\lambda_{C}$, and were understood to mimic the bipolaronic crossover of the exact solution. The asymmetric solutions were furthermore found to be the lowest energy solutions for a large range of parameters. The total energies, and natural occupation numbers, also suggested that the symmetric solution of the fully self-consistent Born approximation describes partially the crossover to a bipolaronic state. In the present work, we studied the frequency structure of the ground state propagators for a restricted range of adiabatic ratios $\gamma=1 / 2,1 / 4$ and interactions $\lambda \in[0,2]$ which allows us to complete some of the observations made in Ref. 1. Firstly, the frequency-integrated observables obtained from the electron propagator are in a better qualitative agreement with exact results than the frequency-resolved objects themselves. In particular, our results show that none of the approximations give an electron propagator in which there is a rigid shift or redistribution of the spectral weight comparable to the exact solution for high $\lambda>1$ interactions. The phonon propagator does not moreover show a clear spectral fingerprint
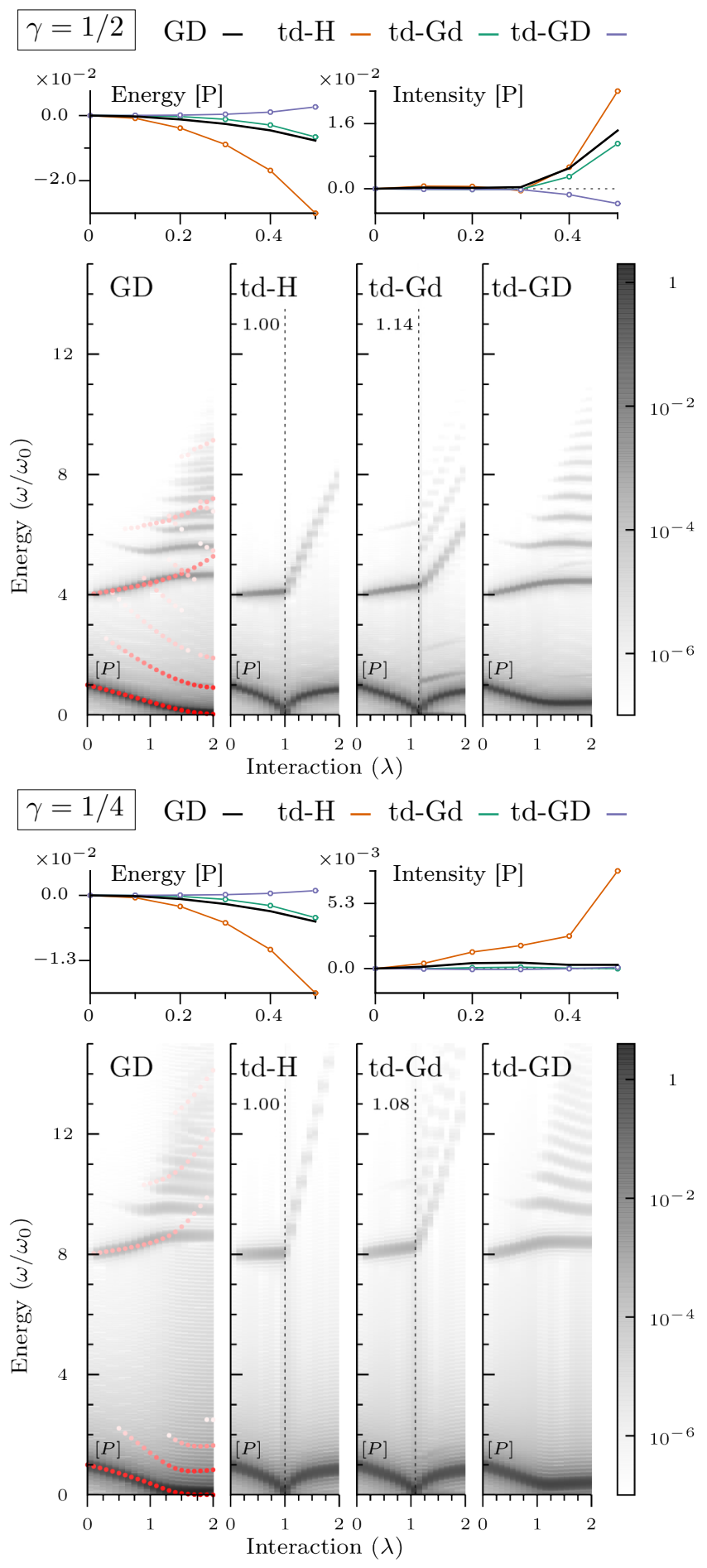

FIG. 12. The phonon propagators as a function of the interaction $\lambda$ and frequency $\omega$. The top and bottom figures correspond to the adiabatic ratios $\gamma=1 / 2$ and $\gamma=1 / 4$, respectively. The contour plots show $\left|D_{11}^{R}(\omega)\right| / T$ for the exact (ED), denoted with red dots, and approximate (GD, td-H, td-Gd, td-GD) solutions. The top panels show the difference between the approximate and exact intensity and position of the lowest energy peak of $\left|D_{11}^{R}(\omega)\right| / T$ labeled with $[P]$ in the contour plots. (color online) 
of a double-well structure in the fully self-consistent Born approximation for the parameters considered. As all of these features have been identified as spectral signatures of a bipolaronic state, our results here favor the statement that none of the approximations describe even partially the bipolaronic crossover for the parameters considered. This said, the results for the electron propagator can be roughly summarized by concluding that the Hartree, partially self-consistent Born, and fully selfconsistent Born approximations agree with the exact results up to very weak $\lambda \ll 1$, weak $\lambda<1$, and weak to intermediate $\lambda \sim 1$ interactions, respectively. The non-interacting phonon propagator used in the Hartree and partially self-consistent Born approximations is only valid for very weak $\lambda \ll 1$ interactions while the dressed propagator of the fully self-consistent Born approximation agrees reasonably well with the exact results up to borderline strong $\lambda \sim 1.5$ interactions.

The linear response functions studied in the present work are obtained by time-propagation starting either from a symmetric or an asymmetric ground state solution. In the case $\lambda>\lambda_{C}$, these solutions co-exits and we find that the symmetric solutions of the Hartree and partially self-consistent Born approximations are unstable, while the asymmetric solutions are stable, against a small asymmetric perturbation. The symmetric and asymmetric solutions of the fully self-consistent Born approximation are, on the other hand, shown to be stable against the same perturbation. By identifying the stable ground state solutions, we have been able to evaluate linear response functions corresponding to these solutions. In particular, the density-density response function obtained in the Hartree and partially self-consistent Born approximations is shown to have a zero-frequency component which appears and its intensity diverges as $\lambda$ approaches $\lambda_{C}$. In the Hartree approximation, this is caused by the build-up of a double-well structure in its ground state energy surface exactly at $\lambda=1$ and by the need of the mean-field to minimize the total energy. Our results further show that the fully self-consistent Born approximation does not have a similar divergence. The comparison of the exact and approximate density-density response functions confirms that the range of validity of the many-body approximations is roughly the same as for the case of equilibrium propagators. In particular, none of the many-body approximations were able to describe the lowest excitation of the exact response function for strong interactions $\lambda>1$ for which it is the dominant feature of the exact response function. By analyzing this excitation, we further identified it as a signature of a bipolaronic system, and hence in agreement with the conclusions based on the equilibrium propagators, this suggests that the approximations do not describe the crossover to the bipolaronic state. Finally, we could by time-propagation obtain another phonon propagator associated with a highly sophisticated self-energy, although a non-selfconsistent one. The results show that although the propagators obtained in the partially and fully self- consistent approximations are better than the equilibrium propagator of the fully self-consistent Born approximation for the perturbative $\lambda \ll 1$ interactions, they lead to deterioration of the qualitative spectral properties for higher interactions. This suggests that, at least in this case, it is either important to maintain self-consistency, or that instead of infinite partial summations of dressed self-energy diagrams it is better to consider truncated approximations.

In order to go beyond the approximations studied here in many-body perturbation theory one needs to introduce vertex corrections which are in particular needed to improve the properties of the electron propagator. This is realizable in an equilibrium theory but leads to a substantial increase in the complexity of the time-domain method which makes the inclusion of vertex corrections challenging with the current computational resources. The two-time equations can be however cast as one-time equations via the $\mathrm{GKBA}^{24-26,34,84-89}$ which is a possible way to overcome the numerical challenge and reduce more advanced approximations tractable. On the other hand e.g. in cavity quantum electrodynamics already our weak interactions are considered strong and thus the approximations used here could be a valuable asset for investigating non-linear time-dependent phenomena. In this context our results provide a basis for studying the approximation in an explicitly time-dependent situation, and for understanding properties of further approximations e.g. the GKBA which could be used in order to address physics of larger or more realistic systems.

\section{ACKNOWLEDGMENTS}

We would like to thank Daniel Karlsson, Riku Tuovinen, and Christian Schäfer for careful reading of the manuscript and useful discussions. We acknowledge CSC IT Center for Science Ltd for the allocation of computational resources. RvL acknowledges the Academy of Finland for support under grant No. 267839.

\section{Appendix A: Hartree, TDSCF and Ehrenfest}

The Hartree approximation is shown here to be equivalent to the time-dependent self-consistent field (TDSCF) approach and to the semi-classical Ehrenfest approximation. This equivalence is shown by deriving the time-dependent self-consistent field equations, noting that they reduce to the Ehrenfest equations of motion, and finally by showing that their solutions can be used to construct the phonon field expectation value, and the electron propagator of the Hartree approximation. We begin by introducing the product ansatz

$$
|\Psi(t)\rangle \equiv|\tilde{\psi}(t)\rangle|\tilde{\chi}(t)\rangle
$$

where $|\tilde{\psi}(t)\rangle$ and $|\tilde{\chi}(t)\rangle$ consist of only electronic and phononic degrees of freedom, respectively. By substitut- 
ing this ansatz to the time-dependent Schrödinger equation

$$
\imath \partial_{t}|\Psi(t)\rangle=\hat{H}(t)|\Psi(t)\rangle
$$

and projecting it to the states $|\tilde{\chi}(t)\rangle$ and $|\tilde{\psi}(t)\rangle$, we arrive at the time-dependent self-consistent field equations ${ }^{90}$

$$
\begin{aligned}
\imath \partial_{t}|\psi(t)\rangle & =\sum_{i j}\left(h_{i j}(t)+\sum_{P} M_{i j}^{P}(t) \phi_{P}(t)\right) \hat{c}_{i}^{\dagger} \hat{c}_{j}|\psi(t)\rangle, \\
\imath \partial_{t}|\chi(t)\rangle & =\left(\sum_{P Q} \Omega_{P Q}(t) \hat{\phi}_{P}(t) \hat{\phi}_{Q}(t)\right. \\
& \left.+\sum_{P} F_{P}(t) \hat{\phi}_{P}(t)+\sum_{i j P} M_{i j}^{P}(t) \gamma_{j i}(t) \hat{\phi}_{P}\right)|\chi(t)\rangle
\end{aligned}
$$

where

$$
\begin{gathered}
\phi_{P}(t) \equiv\left\langle\chi(t)\left|\hat{\phi}_{P}\right| \chi(t)\right\rangle, \\
\gamma_{i j}(t) \equiv\left\langle\psi(t)\left|\hat{c}_{j}^{\dagger} \hat{c}_{i}\right| \psi(t)\right\rangle,
\end{gathered}
$$

are the phonon field expectation value and the reduced density matrix. In the derivation. we adopted the phase conventions

$$
\begin{aligned}
|\psi(t)\rangle & \equiv e^{\imath \int_{t_{0}}^{t} d t^{\prime}\left(E_{p}\left(t^{\prime}\right)-\left\langle\tilde{\chi}\left(t^{\prime}\right) \mid \imath \partial_{t^{\prime}} \tilde{\chi}\left(t^{\prime}\right)\right\rangle\right)}|\tilde{\psi}(t)\rangle, \\
|\chi(t)\rangle & \equiv e^{\imath \int_{t_{0}}^{t} d t^{\prime}\left(E_{e}\left(t^{\prime}\right)-\left\langle\tilde{\psi}\left(t^{\prime}\right) \mid \imath \partial_{t^{\prime}} \tilde{\psi}\left(t^{\prime}\right)\right\rangle\right)}|\tilde{\chi}(t)\rangle,
\end{aligned}
$$

where we defined

$$
\begin{aligned}
& E_{e}(t) \equiv \sum_{i j} h_{i j}(t)\left\langle\psi(t)\left|\hat{c}_{i}^{\dagger} \hat{c}_{j}\right| \psi(t)\right\rangle, \\
& E_{p}(t) \equiv \sum_{P Q} \Omega_{P Q}(t)\left\langle\chi(t)\left|\hat{\phi}_{P} \hat{\phi}_{Q}\right| \chi(t)\right\rangle .
\end{aligned}
$$

as the electron and phonon energies, respectively. The semi-classical Ehrenfest equations are then derived e.g. by introducing a polar expansion of the nuclear state and taking its classical limit ${ }^{90}$. In our case however, the Heisenberg equations of motion for the phonon field expectation values are equal to the classical equations of motion. Taking advantage of this property and the bilinearity of the equation for $|\psi(t)\rangle$ in terms of the electronic operators, we arrive at the Ehrenfest equations of motion

$$
\begin{aligned}
\imath \partial_{t} \psi_{i j}(t) & =\sum_{k}\left(h_{j k}(t)\right. \\
& \left.+\sum_{P} M_{j k}^{P}(t) \phi_{P}(t)\right) \psi_{i k}(t), \\
\imath \sum_{Q} \alpha_{P Q} \partial_{t} \phi_{Q}(t) & =\sum_{Q} \tilde{\Omega}_{P Q}(t) \phi_{Q}(t) \\
& +F_{P}(t)+\sum_{i j} M_{i j}^{P}(t) \gamma_{j i}(t),
\end{aligned}
$$

such that $|\psi(t)\rangle$ can be written as a Slater determinant of the time-dependent orbitals $\psi_{i}(t)$. In order to relate these orbitals to the electron propagator in the Hartree approximation, we further write down the equilibrium Hartree equations

$$
\begin{aligned}
\boldsymbol{h}_{\mathrm{H}}^{M} \vec{\psi}_{k}^{M} & =\epsilon_{k}^{M} \vec{\psi}_{k}^{M}, \\
\boldsymbol{h}_{\mathrm{H}}^{M} & =\boldsymbol{h}^{\mathrm{M}}+\sum_{P} \boldsymbol{M}^{P} \phi_{P}^{M}, \\
\vec{\phi}^{M} & =-\tilde{\boldsymbol{\Omega}}^{M}-1\left(\vec{F}^{\mathrm{M}}+\sum_{i j} \vec{M}_{i j} \gamma_{j i}^{M}\right),
\end{aligned}
$$

which are introduced in Ref. 1, and correspond to a set of non-linear eigenvalue equations for the eigenvalues $\epsilon_{k}^{M}$ and eigenvectors $\psi_{k}^{M}$. The electron propagator can be then written in the Hartree approximation in terms of the time-dependent orbitals $\psi_{i}(t)$ obtained by solving Eqs. (A1) with $\phi_{P}^{M}$ and $\psi_{i}^{M}$ as their initial conditions. That is, in the Hartree approximation, the phonon field expectation values satisfy Eq. (A1b), and the electron propagator is given by

$$
\begin{aligned}
& G_{i j}^{>}\left(t ; t^{\prime}\right)=\frac{1}{\imath} \sum_{k} \bar{f}_{+}\left(\beta \epsilon_{k}^{M}\right) \psi_{k j}^{*}\left(t^{\prime}\right) \psi_{k i}(t), \\
& G_{i j}^{<}\left(t ; t^{\prime}\right)=-\frac{1}{\imath} \sum_{k} f_{+}\left(\beta \epsilon_{k}^{M}\right) \psi_{k j}^{*}\left(t^{\prime}\right) \psi_{k i}(t),
\end{aligned}
$$

where $\bar{f}_{+} \equiv 1-f_{+}$, as readily verified by using Eqs. (A1) to check that Eqs. (5) with the Hartree self-energy are satisfied, and also by verifying that the Kubo-MartinSchwinger boundary conditions ${ }^{2}$ are met.

\section{Appendix B: Hartree Density Response Function}

Here, we calculate the density response function of the two-site, two-electron Holstein model in the Hartree approximation by applying the method discussed in Sec. IV B 1. In order to do this, we first rewrite the Hartree equations in a more convenient form by using the conserved total energy to reduce the number of dependent variables. That is, the total energy of Eq. (22) allows us to eliminate $\Gamma_{1}$, and subsequently by defining the vector

$$
\vec{x} \equiv\left(\begin{array}{c}
n \\
\Gamma_{2} \\
u \\
p
\end{array}\right),
$$

we can rewrite the Hartree equations given in Eqs. (20) as

$$
\begin{aligned}
\dot{\vec{x}} & =\vec{f}(\vec{x}) \\
& \equiv\left(\begin{array}{c}
-t_{\mathrm{kin}} x_{1}+2 g x_{3} \Gamma_{1}\left(x_{1}, x_{3}, x_{4}\right) \\
\omega_{0} x_{4} \\
-\omega_{0} x_{3}+2 g x_{1}
\end{array}\right),
\end{aligned}
$$


where $8 t_{\text {kin }} \Gamma_{1}\left(x_{1}, x_{3}, x_{4}\right) \equiv \omega_{0}\left(x_{4}^{2}+x_{3}^{2}-1\right)-4 g x_{1} x_{3}-$ $2 E_{0}$. Here $E_{0}$ denotes the total energy at $t=0$ which is determined by the initial condition $\vec{x}^{0}$. The densitydensity response function of Eq. (16), which is in this case given by

$$
\chi_{i j}^{R}(t)=\left.(-1)^{i+1} \frac{\partial n(t)}{\partial v_{j}}\right|_{v=0} .
$$

is then the $i=1$ component of the more general response function $\partial x_{i} /\left.\partial v_{j}\right|_{v=0}$ which satisfies

$$
\left.\frac{d}{d t} \frac{\partial \vec{x}}{\partial v_{j}}\right|_{v=0}=\left.\boldsymbol{J} \frac{\partial \vec{x}}{\partial v_{j}}\right|_{v=0}
$$

as seen by differentiating Eq. (B1) with respect to $v_{j}$. The Jacobian matrix $\left.J_{i j} \equiv \partial_{x_{j}} f_{i}(\vec{x})\right|_{v=0}$ is a function of the unperturbed solution $\left.\vec{x}\right|_{v=0}$ which is in our case obtained by propagating either the symmetric or asymmetric ground state solution of the equilibrium Hartree equations given in Eq. (21a) and Eq. (21b), respectively. As these solutions are also fixed-points of Eq. (B1), they are constant in time, and thus the Jacobian matrices for the symmetric $(s)$ and both asymmetric $(a)$ solutions given by

$$
\begin{aligned}
& \boldsymbol{J}_{s} \equiv\left(\begin{array}{cccc}
0 & 4 t_{\mathrm{kin}} & 0 & 0 \\
-t_{\mathrm{kin}} & 0 & g & 0 \\
0 & 0 & 0 & \omega_{0} \\
2 g & 0 & -\omega_{0} & 0
\end{array}\right) \\
& \boldsymbol{J}_{a} \equiv\left(\begin{array}{cccc}
0 & 4 t_{\mathrm{kin}} & 0 & 0 \\
-t_{\mathrm{kin}} \lambda^{2} & 0 & g \lambda^{-1} & 0 \\
0 & 0 & 0 & \omega_{0} \\
2 g & 0 & -\omega_{0} & 0
\end{array}\right)
\end{aligned}
$$

are time-independent. The initial conditions $\partial \vec{x}_{\eta}^{0} /\left.\partial v_{j}\right|_{v=0}$ for the symmetric $(\eta=s)$ and asymmetric $(\eta=a)$ cases can be deduced from Eqs. (18) to be

$$
\begin{aligned}
& \left.\frac{\partial \vec{x}_{s}^{0}}{\partial v_{j}}\right|_{v=0}=\left(\begin{array}{c}
0 \\
-\left(\delta_{1 j}-\delta_{2 j}\right) / 2 \\
0 \\
0
\end{array}\right) . \\
& \left.\frac{\partial \vec{x}_{a}^{0}}{\partial v_{j}}\right|_{v=0}=\left(\begin{array}{c}
-\left(\delta_{1 j}-\delta_{2 j}\right) \lambda^{-1} / 4 \\
0 \\
0
\end{array}\right) .
\end{aligned}
$$

respectively. The equation for the response function is linear, and hence admits the solution

$$
\left.\frac{\partial \vec{x}_{\eta}(t)}{\partial v_{j}}\right|_{v=0}=\left.e^{\boldsymbol{J}_{\eta} t} \frac{d \vec{x}_{\eta}^{0}}{d v_{j}}\right|_{v=0}
$$

where we restored the explicit time-dependence. The task is then to evaluate the matrix exponential which is done here by using the eigendecomposition of the Ja- cobian matrix. The decomposition exists since the eigenvalues of the Jacobian matrix given by $\imath \omega_{ \pm}^{\eta},-\imath \omega_{ \pm}^{\eta}$, where

$$
\begin{aligned}
& \omega_{ \pm}^{s} \equiv \sqrt{\frac{\omega_{0}^{2}+4 t_{\mathrm{kin}}^{2}}{2}\left(1 \pm \sqrt{\left.1+\frac{16 \omega_{0}^{2} t_{\mathrm{kin}}^{2}(\lambda-1)}{\left(\omega_{0}^{2}+4 t_{\mathrm{kin}}^{2}\right)^{2}}\right)}\right.}, \\
& \omega_{ \pm}^{a} \equiv \sqrt{\frac{\omega_{0}^{2}+4 t_{\mathrm{kin}}^{2} \lambda^{2}}{2}\left(1 \pm \sqrt{1-\frac{16 \omega_{0}^{2} t_{\mathrm{kin}}^{2}\left(\lambda^{2}-1\right)}{\left(\omega_{0}^{2}+4 t_{\mathrm{kin}}^{2} \lambda^{2}\right)^{2}}}\right)},
\end{aligned}
$$

are non-degenerate for $\lambda \neq 1$. The diagonalizing similarity transformation given by

$$
\boldsymbol{X}^{\eta} \equiv\left(\begin{array}{cccc}
1 & 1 & 1 & 1 \\
\imath \omega_{+}^{\eta} / 4 t_{\mathrm{kin}} & \imath \omega_{-}^{\eta} / 4 t_{\mathrm{kin}} & -\imath \omega_{+}^{\eta} / 4 t_{\mathrm{kin}} & -\imath \omega_{-}^{\eta} / 4 t_{\mathrm{kin}} \\
x_{+}^{\eta} & x_{-}^{\eta} & x_{+}^{\eta} & x_{\overline{-}}^{\eta} \\
\imath \omega_{+}^{\eta} x_{+}^{\eta} & \imath \omega_{-}^{\eta} x_{-}^{\eta} & -\imath \omega_{+}^{\eta} x_{+}^{\eta} & -\imath \omega_{-}^{\eta} x_{-}^{\eta}
\end{array}\right)
$$

where $x_{ \pm}^{\eta} \equiv 2 g /\left(1-\omega_{ \pm}^{\eta} 2\right)$, then allows us to write the response function as

$$
\left.\frac{\partial \vec{x}_{\eta}(t)}{\partial v_{j}}\right|_{v=0}=\left.\boldsymbol{X} e^{\imath \boldsymbol{\omega}^{\eta} t} \boldsymbol{X}^{-1} \frac{\partial \vec{x}_{\eta}^{0}}{\partial v_{j}}\right|_{v=0},
$$

where $\boldsymbol{\omega}^{\eta}=\operatorname{diag}\left(\omega_{+}^{\eta}, \omega_{-}^{\eta},-\omega_{+}^{\eta},-\omega_{-}^{\eta}\right)$ denotes a diagonal matrix. In particular, its first component according to Eq. (B2) gives the density response function

$$
\begin{aligned}
\chi_{\mathrm{H}_{\eta} ; i j}^{R}(t) & =-(-1)^{i-j} \theta(t)\left(\chi_{+}^{\eta} \sin \left(\omega_{+}^{\eta} t\right)+\chi_{-}^{\eta} \sin \left(\omega_{-}^{\eta} t\right)\right), \\
\chi_{ \pm}^{\eta} & \equiv \frac{2 t_{\mathrm{kin}}\left(\omega_{0}^{2}-\omega_{ \pm}^{\eta 2}\right)^{2}}{\omega_{ \pm}^{\eta}\left[\left(\omega_{0}^{2}-\omega_{ \pm}^{\eta}\right)^{2}+4 \lambda \omega_{0}^{2} t_{\mathrm{kin}}^{2}\right]},
\end{aligned}
$$

where we introduced the Heaviside function to enforce the correct causal structure. 
${ }^{1}$ N. Säkkinen, Y. Peng, H. Appel, and R. van Leeuwen, In preparation.

${ }^{2}$ G. Stefanucci and R. van Leeuwen, Nonequilibrium Many-Body Theory of Quantum Systems: A Modern Introduction (Cambridge, 2013).

${ }^{3}$ M. Galperin, M. A. Ratner, and A. Nitzan, J. Phys.: Condens. Matter 19, 103201 (2007).

${ }^{4}$ K. S. Thygesen and A. Rubio, Phys. Rev. B 77, 115333 (2008). ${ }^{5}$ X. Wang, C. D. Spataru, M. S. Hybertsen, and A. J. Millis, Phys. Rev. B 77, 045119 (2008).

${ }^{6}$ C. D. Spataru, M. S. Hybertsen, S. G. Louie, and A. J. Millis, Phys. Rev. B 79, 155110 (2009).

${ }^{7}$ S. Schmitt and F. B. Anders, Phys. Rev. B 81, 165106 (2010).

${ }^{8}$ P. Myöhänen, A. Stan, G. Stefanucci, and R. van Leeuwen, Europhys. Lett. 84, 67001 (2008).

${ }^{9}$ P. Myöhänen, A. Stan, G. Stefanucci, and R. van Leeuwen, Phys. Rev. B 80, 115107 (2009).

${ }^{10}$ A.-M. Uimonen, E. Khosravi, G. Stefanucci, S. Kurth, R. van Leeuwen, and E. K. U. Gross, Journal of Physics: Conference Series 220, 012018 (2010).

${ }^{11}$ A.-M. Uimonen, E. Khosravi, A. Stan, G. Stefanucci, S. Kurth, R. van Leeuwen, and E. K. U. Gross, Phys. Rev. B 84, 115103 (2011).

${ }^{12}$ P. Myöhänen, R. Tuovinen, T. Korhonen, G. Stefanucci, and R. van Leeuwen, Phys. Rev. B 85, 075105 (2012).

${ }^{13}$ E. Khosravi, A.-M. Uimonen, A. Stan, G. Stefanucci, S. Kurth, R. van Leeuwen, and E. K. U. Gross, Phys. Rev. B 85, 075103 (2012).

${ }^{14}$ M. Galperin, M. A. Ratner, and A. Nitzan, Nano Letters 5, 125 (2005), pMID: 15792425, http://dx.doi.org/10.1021/nl048216c.

${ }^{15}$ A. Mitra, I. Aleiner, and A. J. Millis, Phys. Rev. Lett. 94, 076404 (2005).

${ }^{16}$ A. S. Alexandrov and A. M. Bratkovsky, Journal of Physics: Condensed Matter 19, 255203 (2007).

${ }^{17}$ M. Galperin, A. Nizzan, and M. A. Ratner, J. Phys.: Condens. Matter 20, 374107 (2008).

${ }^{18}$ A. S. Alexandrov and A. M. Bratkovsky, Phys. Rev. B 80, 115321 (2009).

${ }^{19}$ A. A. Dzhioev and D. S. Kosov, The Journal of Chemical Physics 135, 174111 (2011).

${ }^{20}$ K. F. Albrecht, H. Wang, L. M. Uhlbacher, M. Thoss, and A. Komnik, Phys. Rev. B 86, 081412 (2012).

${ }^{21}$ E. Y. Wilner, H. Wang, G. Cohen, M. Thoss, and E. Rabani, Phys. Rev. B 88, 045137 (2013).

${ }^{22}$ E. Y. Wilner, H. Wang, M. Thoss, and E. Rabani, "Nonequilibrium dynamics with electron-phonon interactions: Transient dynamics and approach to steady state," (2014), arXiv:condmat.str-el/1402.6454.

${ }^{23}$ E. Y. Wilner, H. Wang, M. Thoss, and E. Rabani, Phys. Rev. B 90, 115145 (2014).

${ }^{24}$ A. Marini, J. Phys: Conf. Proc. 427, 012003 (2013).

${ }^{25}$ D. Sangalli and A. Marini, EPL (Europhysics Letters) 110, 47004 (2015).

${ }^{26}$ D. Sangalli and A. Marini, Journal of Physics: Conference Series 609, 012006 (2015).

${ }^{27}$ P. Lipavský, V. Špička, and B. Velický, Phys. Rev. B 34, 6933 (1986).

${ }^{28}$ L. S. Cederbaum and W. Domcke, The Journal of Chemical Physics 60, 2878 (1974).

${ }^{29}$ M. Ruggenthaler, J. Flick, C. Pellegrini, H. Appel, I. V. Tokatly, and A. Rubio, Phys. Rev. A 90, 012508 (2014).

${ }^{30}$ C. Pellegrini, J. Flick, I. Tokatly, Appel.H., and A. Rubio, "Optimized Effective Potential for Quantum Electrodynamical TimeDependent Density Functional Theory," (2014), arXiv:1412.4530 [quant-ph].

${ }^{31}$ N. E. Dahlen and R. van Leeuwen, Phys. Rev. Lett. 98, 153004 (2007).

${ }^{32}$ A. Stan, N. E. Dahlen, and R. van Leeuwen, J. Chem. Phys. 130, 224101 (2009).
${ }^{33}$ L. P. Kadanoff and G. Baym, Quantum Statistical Mechanics (New York: Benjamin, 1962).

${ }^{34}$ P. Gartner, J. Seebeck, and F. Jahnke, Phys. Rev. B 73, 115307 (2006).

${ }^{35}$ G. Baym and L. P. Kadanoff, Phys. Rev. 124, 287 (1961).

${ }^{36}$ G. Baym, Phys. Rev. 127, 1391 (1962).

${ }^{37}$ N.-H. Kwong and M. Bonitz, Phys. Rev. Lett. 84, 1768 (2000).

${ }^{38}$ N. Säkkinen, M. Manninen, and R. van Leeuwen, New. J. Phys. 14, 0132032 (2012).

${ }^{39}$ K. Balzer, S. Hermanns, and M. Bonitz, EPL (Europhysics Letters) 98, 67002 (2012).

${ }^{40}$ G. Onida, L. Reining, and A. Rubio, Rev. Mod. Phys. 74, 601 (2002).

${ }^{41}$ P. Romaniello, D. Sangalli, J. A. Berger, F. Sottile, L. G. Molinari, L. Reining, and G. Onida, The Journal of Chemical Physics 130, 044108 (2009).

${ }^{42}$ D. Sangalli, P. Romaniello, G. Onida, and A. Marini, The Journal of Chemical Physics 134, 034115 (2011).

${ }^{43}$ D. Zhang, S. N. Steinmann, and W. Yang, The Journal of Chemical Physics 139, 154109 (2013).

${ }^{44}$ I. I. Rabi, Phys. Rev. 49, 324 (1936).

${ }^{45}$ I. I. Rabi, Phys. Rev. 51, 652 (1937).

${ }^{46}$ B. I. Lundqvist, Phys. kondens. Materie 6, 193 (1967).

${ }^{47}$ T. Holstein, Ann. Phys. 281, 706724 (2000).

${ }^{48}$ R. H. Dicke, Phys. Rev. 93, 99 (1954).

${ }^{49}$ K. Hepp and E. Lieb, Ann. Phys. 76, 360 (1973).

${ }^{50}$ Y. K. Wang and F. T. Hioe, Phys. Rev. A 7, 831 (1973).

${ }^{51}$ C. Emary and T. Brandes, Phys. Rev. Lett. 90, 044101 (2003).

${ }^{52}$ G. Levine and W. P. Su, Phys. Rev. B 43, 10413 (1991).

${ }^{53}$ F. Marsiglio, Phys. Rev. B 42, 2416 (1990).

${ }^{54}$ J. K. Freericks, M. Jarrell, and D. J. Scalapino, Phys. Rev. B 48, 6302 (1993).

${ }^{55}$ J. K. Freericks, Phys. Rev. B 50, 403 (1994).

${ }^{56}$ L. Hedin, Phys. Rev. 139, A796 (1965).

${ }^{57}$ W. Schmidt and M. Schreiber, J. Chem. Phys. 86, 953 (1987).

${ }^{58}$ D. Feinberg, S. Ciuchi, and F. de Pasquale, Int. J. Mod. Phys. B 04, 1317 (1990).

${ }^{59}$ J. Ranninger and U. Thibblin, Phys. Rev. B 45, 1991 (1992).

${ }^{60}$ A. S. Alexandrov, V. V. Kabanov, and D. K. Ray, Phys. Rev. B 49, 9915 (1994).

${ }^{61}$ F. Marsiglio, Physica C 244, 21 (1995).

${ }^{62}$ E. V. L. de Mello and J. Ranninger, Phys. Rev. B 55, 14872 (1997).

63Ž. Crljen, Fizika A 7, 75 (1998).

${ }^{64}$ H. Rongsheng, L. Zijing, and W. Kelin, Phys. Rev. B 65, 174303 (2002).

${ }^{65}$ R. Qing-Bao and C. Qing-Hu, Comm. Theor. Phys. 43, 357 (2005).

${ }^{66}$ S. Paganelli and S. Ciuchi, Eur. Phys. J. Special Topics 160, 343 (2008).

${ }^{67}$ S. Paganelli and S. Cuichi, J. Phys. Condensed Matter 20, 235203 (2008).

${ }^{68}$ Y. A. Firsov and E. K. Kudinov, Phys. Solid State 39, 1930 (1997).

${ }^{69}$ J. Chatterjee and A. N. Das, Phys. Rev. B 61, 4592 (2000).

${ }^{70}$ E. O. Brigham, The Fast Fourier Transform (Prentice-Hall Inc., 1974).

${ }^{71}$ M. Capone and S. Ciuchi, Phys. Rev. Lett. 91, 186405 (2003).

${ }^{72}$ B. Holm and U. von Barth, Phys. Rev. B 57, 2108 (1998).

${ }^{73}$ M. W. Hirsch and S. Smale, Differential Equations, Dynamical Systems, and Linear Algebra (Academic Press Inc., 1974).

${ }^{74}$ L. Perko, Differential Equations and Dynamical Systems, 3rd ed. (Springer-Verlag, New York Inc., 2001).

${ }^{75}$ D. W. Jordan and P. Smith, Nonlinear Ordinary Differential Equations: and introduction for scientists and engineers, 4th ed. (Oxford University Press, 2007).

${ }^{76}$ M. A. M. de Aguiar, K. Furuya, and M. C. Nemes, Quantum Optics: Journal of the European Optical Society Part B 3, 305 (1991). 
${ }^{77}$ A. P. Itin and P. Törmä, "Dynamics of quantum phase transitions in dicke and lipkin-meshkov-glick models," (2010), arXiv:0901.4778v3 [cond-mat.stat-mech].

${ }^{78}$ L. Bakemeier, A. Alvermann, and H. Fehske, Phys. Rev. A 88, 043835 (2013).

${ }^{79}$ V. I. Arnold, V. V. Kozlov, and A. I. Neihstadt, Mathematical Aspects of Classical and Celestial Mechanics (Spinger-Verlag Berlin Heidelberg, 2006).

${ }^{80}$ R. Krechetnikov and J. E. Marsden, Rev. Mod. Phys. 79, 519 (2007).

${ }^{81}$ Y. Kuznetsov, Elements of applied bifurcation theory, 2nd edition (Springer, New York, 1998).

82 A.-M. Uimonen, G. Stefanucci, Y. Pavlyukh, and R. van Leeuwen, Phys. Rev. B 91, 115104 (2015).

${ }^{83} \mathrm{R}$. van Leeuwen and N. E. Dahlen, in The Electron Liquid Paradigm in Condensed Matter Physics, Proc. of the International School of Physics Enrico Fermi, Vol. CLVII (Amsterdam:
IOS Press, 2004)

${ }^{84}$ H. S. Köhler, Phys. Rev. E 53, 3145 (1996).

${ }^{85}$ M. Bonitz, D. Kremp, D. C. Scott, R. Binder, W. D. Kraeft, and H. S. Khler, Journal of Physics: Condensed Matter 8, 6057 (1996).

${ }^{86}$ G. Pal, Y. Pavlyukh, H. C. Schneider, and W. Hübner, Eur. Phys. J. B 70, 483 (2009).

${ }^{87}$ G. Pal, Y. Pavlyukh, W. Hübner, and H. C. Schneider, Eur. Phys. J. B 79, 327 (2011).

${ }^{88}$ K. Balzer, S. Hermanns, and M. Bonitz, J. Phys: Conf. Proc. 427, 012006 (2013).

${ }^{89}$ S. Latini, E. Perfetto, A.-M. Uimonen, R. van Leeuwen, and G. Stefanucci, Phys. Rev. B 89, 075306 (2014).

${ }^{90}$ D. Marx and J. Hutter, Ab Initio Molecular Dynamics: basic theory and advanced methods (Cambridge University Press, 2009). 